\title{
Coverage Analysis of mmWave and THz-enabled Aerial and Terrestrial Heterogeneous Networks
}

\author{
Adil Ali Raja, Haris Pervaiz, Syed Ali Hassan, Sahil Garg, Md. Jalil Piran, M. Shamim Hossain
}

\begin{abstract}
Heterogeneous networks (HetNets) are becoming a promising solution for future wireless systems to satisfy the high data rate requirements. This paper introduces a stochastic geometry framework for the analysis of the downlink coverage probability in a multi-tier HetNet consisting of macro-base station (MBS) operating at sub-6 GHz, millimeter wave (mmWave)enabled unmanned aerial vehicles (UAVs) operating at $28 \mathrm{GHz}$ and small BSs operating both at mmWave and $\mathrm{THz}$ frequencies. The analytical expressions for the coverage probability for each tier have been derived in the paper, whereas Monte Carlo simulations are then performed to validate the analytical expressions. The effectiveness of the HetNet is analyzed on various performance metrics including association and coverage probabilities for different network parameters. It has been shown that the mmWave and THz-enabled cells provide significant improvement in the achievable data rates because of their high available bandwidths, however, they have a degrading effect on the coverage probability due to their high propagation losses.
\end{abstract}

Index Terms-UAVs, millimeter wave, TeraHertz, SINR coverage probability.

\section{INTRODUCTION}

$\mathbf{T}$ RADITIONAL base stations (BSs) due to their low frequency bands (sub-6 GHz) and limited bandwidth are unable to cope with very high demand for data rates. This demand raises the need to have small cells operating at high frequencies such as millimeter wave (mmWave) and TeraHertz (THz) frequencies [1], [2]. On the other hand, the need for infrastructure-less networks such as unmanned aerial vehicles (UAVs) also gained importance as they can be utilized to serve over-crowded places or regions where terrestrial networks are unable to provide coverage [3]. Each above mentioned technology has particular associated characteristics with it. Characteristics such as availability of high bandwidth at mmWave and $\mathrm{THz}$ frequencies significantly improve the performance of the network by providing very high data rates as compared to sub- $6 \mathrm{GHz}$ frequency band. However, these technologies also pose several challenges such as very high propagation losses. At $\mathrm{THz}$ frequencies, these losses become

A. A. Raja and S. A. Hassan are with the School of Electrical Engineering and Computer Science (SEECS), National University of Sciences and Technology (NUST), Islamabad 44000, Pakistan. E-mail: \{araja.phd15seecs,ali.hassan\}@ seecs.edu.pk

H. Pervaiz is with the School of Computing and Communications, Lancaster University, Lancaster LA1 4YW, U.K. E-mail: h.b.pervaiz@lancaster.ac.uk

S. Garg is with the Electrical Engineering Department, École de technologie supérieure, Montréal, QC H3C 1K3, Canada. E-mail: sahil.garg@ieee.org

Md. Jalil Piran is with the Department of Computer Science and Engineering, Sejong University, Seoul 05006, South Korea E-mail: piran@sejong.ac.kr

M. Shamim Hossain is with the Department of Software Engineering, College of Computer and Information Sciences, King Saud University, Riyadh 11543, Saudi Arabia. E-mail: mshossain@ksu.edu.sa more severe because of the high molecular absorption. Thus there is a trade off between high data rate and high propagation losses at such high frequencies. The integration of the above mentioned technologies in the form of heterogeneous networks (HetNets) has now become the focus of research to meet the ever increasing demand of ultra high data rates.

Recently, the use of $\mathrm{THz}$ band to achieve high data rates has been explored in various studies. In [4], the authors surveyed the challenges in $\mathrm{THz}$ communication and provided an indepth analysis of THz networks. In [5], the authors discussed the problems associated with distance limitation at $\mathrm{THz}$ frequencies. The molecular absorption losses vary significantly with the choice of frequency windows in $\mathrm{THz}$ band. In [6], the authors studied the impact of various $\mathrm{THz}$ frequency windows for $\mathrm{THz}$ communication. Efficient deployment of $\mathrm{THz}$ communication systems is needed in order to enjoy the benefits of $\mathrm{THz}$ frequency band. For that purpose in [7], several features of $\mathrm{THz}$ communication have been studied to improve its deployment. In [8], the authors proposed a hybrid mmWave-THz enabled Internet-of-things (IoT) network and derived closed-form expressions to evaluate the spectral efficiency and coverage probability of the network. They found that the network performance significantly improves by the use of $\mathrm{THz}$ base stations.

The rise in HetNet deployment raises the need for analytical frameworks to perform coverage and rate analysis [9]. In [10], the authors performed the coverage and rate analysis on a device-to-device (D2D) and UAV communication network. The authors in [11]-[14] provided analytical frameworks for coverage analysis of UAV-aided mmWave wireless networks. In [15], the authors derived the coverage probability for a hybrid $\mathrm{THz}$ and radio frequency (RF) wireless network. The authors in [16] computed the coverage probability in a network, where BSs can either operate on $\mathrm{THz}$ or sub- $6 \mathrm{GHz}$ frequencies. In [17], the authors considered an interference regime in a $\mathrm{THz}$ only network and computed the mean interference.

The capabilities of UAVs for infrastructure-less and rapid deployment in an overly crowded environment or disaster hit areas make the UAVs an essential part of any HetNet [18]. UAVs have the ability to change their altitude as opposed to terrestrial BSs. In [19], the authors provided a geometric lineof-sight (LoS) model for UAV and also depicted the optimal UAV altitude for maximum coverage probability. In [20], the authors computed the UAVs optimal position for backhauling the data between small cells and the core network. The authors in [21] studied and analyzed various quality-of-service (QoS) metrics for a coexisting network of sub- $6 \mathrm{GHz}$ and mmWave 
UAVs. Numerous studies in analytical framework for coverage analysis of elevated base stations have been conducted in [22]-[24]. A lot of work has also been done in mmWaveaided networks. In [25], the authors provided an analysis for rate, coverage and energy efficiency using tools from stochastic geometry in a mmWave-aided multi-tier network. In [26], physical layer security has been analyzed in a massive multiple-input multiple-output (MIMO) HetNet. The coverage and rate analysis for mmWave networks has been extensively studied in [27]-[29]. In [30], mmWave channel models and their closed-form expressions are derived for different communication scenarios. The effects of hovering UAV fluctuations and antenna directivity gains on the outage probability are also analyzed in the study. A similar approach for hover time optimization is studied in [31]. A novel sectoring approach to cover entire area while using mmWave antenna array is presented in [32].

To our best knowledge, this is the first contribution that presented an extensive analytical framework based on tools from stochastic analysis for coverage and rate analysis in a HetNet comprising of macro base station (MBS) operating on sub-6 $\mathrm{GHz}$, UAVs and small cells operating on mmWave frequency and THz-enabled small cells. The main contributions can be summarized as follows.

- For the proposed multi-tier hybrid HetNet, an analytical tractable framework has been developed to compute association and coverage probabilities with the aid of stochastic geometry. Our results show that the users associated with mmWave and $\mathrm{THz}$ frequencies-enabled cells achieve very high data rates because of a larger available bandwidth but the high propagation losses encountered at these frequencies have a degrading effect on SINR coverage probability.

- We have also shown the effect of sub-6 GHz UAVs and mmWave UAVs on the SINR and rate coverage probabilities by changing the proportion of sub- $6 \mathrm{GHz}$ UAVs with respect to mmWave UAVs in the HetNet. The effect of biasness and cell densities on SINR and rate coverage probabilities have also been depicted in our work.

The rest of the paper is organized as follows. Section II introduces the system model. Section III provides expressions for association and coverage probabilities. Section IV contains detailed simulation results. In Section $\mathrm{V}$, conclusions are drawn.

\section{SySTEM MODEL}

We consider a multi-tier HetNet as shown in Fig. 1. The tier 1 consists of sub- $6 \mathrm{GHz}$ MBSs, tier 2 is composed of mmWave small cells (MSC), tier 3 comprises of $\mathrm{THz}$ small cells (TSC) and tier 4 constitutes mmWave UAVs. Independent homogeneous Poisson point processes (HPPP), $\phi_{i}$, are used to model BS locations having densities $\lambda_{i}$ where $i \in\{1,2,3,4\}$ for MBS, MSC, TSC and UAV, respectively. It is assumed that all the BSs of a particular tier have same transmission power. The user locations also follow an independent homogeneous PPP, $\phi_{u}$, with density $\lambda_{u}$. Each user measures the channel

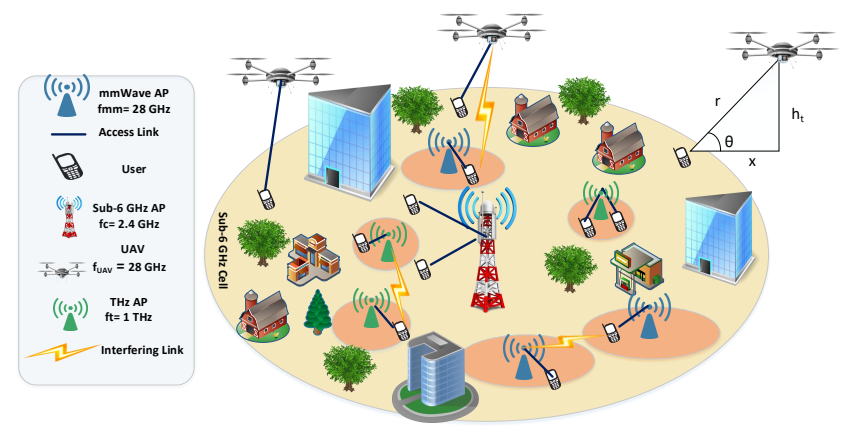

Fig. 1: A graphical snapshot of integrated aerial and terrestrial heterogeneous network consisting of MBS operating at sub$6 \mathrm{GHz}$ band with density $\lambda_{1}=4 \times 10^{-6} \mathrm{BS} / \mathrm{m}^{2}$, mmWaveenabled access points with density $\lambda_{2}=3 \times \lambda_{1} \mathrm{BS} / \mathrm{m}^{2}$ and THz-enabled access points with density $\lambda_{3}=3 \times \lambda_{1} \mathrm{BS} / \mathrm{m}^{2}$ supported by aerial base stations with density $\lambda_{4}=3 \times$ $\lambda_{1} \mathrm{BS} / \mathrm{m}^{2}$ in area with cellular radius $=500$ meters .

quality and associates with the BS providing maximum biased average received power. The envelope of the fading, $|h|$, between a transmitter and a receiver, follows a Nakagami fading model and its probability density function (PDF) is as follows

$$
|h| \sim f_{|h|}\left(l, \Omega_{w}\right) \triangleq \frac{\Omega_{w}^{\Omega_{w}} l^{\Omega_{w}-1} \exp \left\{-\Omega_{w} l\right\}}{\gamma\left(\Omega_{w}\right)}, \forall l>0,
$$

where $\Omega_{w}$ is the Nakagami fading parameter wherein $w \in$ $\left\{l_{m}, n_{m}\right\}$ and $\gamma($.$) is the gamma function. \Omega_{l_{m}}$ and $\Omega_{n_{m}}$ are Nakagami fading parameters where LoS and NLoS propagation environments are represented by $l_{m}$ and $n_{m}$, respectively. The Nakagami parameter for each environment is differently characterized. The channel for sub- $6 \mathrm{GHz}$ frequencies is assumed to be Rayleigh fading, i.e., Nakagami fading with $\Omega_{w}=1$.

In our work, maximum biased received power is considered for user association with a BS. The association scheme determines the tier $k$ for a typical user such that

$$
k=\arg \max _{i \in\{1,2,3,4\}}\left(\widetilde{P}_{i} G_{i} x^{-\beta_{i}}\right)
$$

where $\widetilde{P}_{i}=P_{i} /\left(4 \pi f_{c, i} / c\right)^{2}$ is the normalized power of $i^{\text {th }}$ tier, $G_{i}$ denotes the antenna gains for tier $i, x$ represents the distance between the typical user and the BS of tier $i, \beta_{i}$ is the path loss exponent of tier $i, f_{c, i}$ is the carrier frequency of that particular tier and $c$ is the speed of light. In (2), $P_{i}=\psi_{i} \times \bar{P}_{i}$, where $\psi_{i}$ is the bias value of $i^{t h}$ tier and $\bar{P}_{i}$ is the transmission power of tier $i$. We now discuss the system model of each tier.

\section{A. Sub-6 GHz MBS tier}

The received power at a typical user in tier 1 (i.e. MBS) is given as

$$
P_{r, \mathrm{MBS}}[d B]=P_{1}-P L_{\mathrm{MBS}},
$$


where $P_{1}$ is the biased transmitted power and $P L_{\mathrm{MBS}}$ is the path loss of tier 1. $P L_{\mathrm{MBS}}$ can be evaluated as

$$
P L_{\mathrm{MBS}}=20 \log \left(\frac{4 \pi f_{c, 1}}{c}\right)+10 \beta_{1} \log (x),
$$

where $f_{c, 1}$ represents the carrier frequency, $x$ denotes the distance between the transmitter and the receiver and $\beta_{1}$ is the path loss exponent. The signal-to-interference plus noise ratio (SINR) experienced by a typical user is given as

$$
\mathrm{SINR}_{\mathrm{MBS}}=\frac{P_{r, \mathrm{MBS}}}{\sigma_{1}^{2}+I_{1}}
$$

where $\sigma_{1}^{2}$ denotes the power spectral density of the noise and $I_{1}=\sum_{k \in \phi_{1} /\left\{a_{o}\right\}} P_{1} h_{k} x_{k}^{-\beta_{1}}$ is the cumulative interference from other BSs in tier 1.

\section{B. mmWave Small Cell tier}

The mmWave small cells are distributed using a PPP and because of the blockages in mmWave band, this distribution can be further divided into two independent non-homogeneous PPPs, $\phi_{m m, L}$ and $\phi_{m m, N}$ for LoS and NLoS mmWave small cells, respectively, by using independent thinning theorem. For analytical tractability, a typical user is assumed to be located at origin $O$. The user is considered in LoS to MSC BS located at $T$ if there is no blockage in the path $O T . \phi_{m m, L}$ and $\phi_{m m, N}$ have the densities $\mathfrak{P}_{L o S}(x) \lambda_{2}$ and $\left(1-\mathfrak{P}_{L o S}(x)\right) \lambda_{2}$, where $\mathfrak{P}_{L o S}(x)$ is the LoS probability function. The function $\mathfrak{P}_{L o S}(x)$ can be evaluated using blockage models from stochastic geometry or from field measurements and is given by $e^{-\varphi x}$ where $\varphi$ is the environment dependent variable and $x$ is the distance between transmitter and the receiver.

The MSC tier incorporates directional beamforming to compensate for the pathloss at mmWave frequencies. For MSC tier, the SINR experienced by a typical user connected to serving MSC $b_{o}$ is given by

$$
\operatorname{SINR}_{\mathrm{MSC}}=\frac{\overbrace{\bar{P}_{2} \psi_{2}}^{\tilde{P}_{2}} G_{r}^{m m} G_{t}^{m m} h_{x} x^{-\beta_{2}}}{\sum_{i \in(l, n)} \sum_{j \in\left\{\phi_{m m} / b_{o} \cup \phi_{U A V}\right\}} P_{2} G_{V} h_{j} x_{j}^{-\beta_{2 i}}+\sigma_{2}^{2}},
$$

where $l$ and $n$ represents the LoS and NLoS interfering links, $G_{r}^{m m}$ and $G_{t}^{m m}$ are the receiver and transmitter antennas main lobe gains, respectively, $h_{x}$ is the Nakagami fading, $x$ denotes the distance between the user and the MSC, $\beta_{2}$ is the path loss exponent, $\sigma_{2}^{2}$ is the noise power spectral density and $\psi_{2}$ is the bias factor. The directivity gain of the interfering antenna is given by $G_{V}^{m m}$. Both BSs and users are assumed to be in perfect alignment so the desired link directivity gain is given by $G_{r}^{m m} G_{t}^{m m}$. The directivity gain, $G_{V}^{m m}$, where $V \in\{1,2,3,4\}$ is given as,

$$
G_{V}^{m m}=\left\{\begin{array}{ll}
G_{1}=G_{r}^{m m} G_{t}^{m m}, & \text { with prob. } \frac{\Theta_{r} \Theta_{t}}{4 \pi^{2}} \\
G_{2}=G_{r}^{m m} g_{t}^{m m}, & \text { with prob. } \frac{\Theta_{r}\left(2 \pi-\Theta_{t}\right)}{4 \pi^{2}} \\
G_{3}=g_{r}^{m m} G_{t}^{m m}, & \text { with prob. } \frac{\left(2 \pi-\Theta_{r}\right) \Theta_{t}}{4 \pi^{2}} \\
G_{4}=g_{r}^{m m} g_{t}^{m m}, & \text { with prob. } \frac{\left(2 \pi-\Theta_{r}\right)\left(2 \pi-\Theta_{t}\right)}{4 \pi^{2}}
\end{array},\right.
$$

where $g_{t}^{m m}$ and $g_{r}^{m m}$ are side lobe gains for transmitter and receiver, and the half power beamwidths of transmitter and receiver are given by $\Theta_{t}$ and $\Theta_{r}$.

\section{C. $\mathrm{THz}$ Small Cell tier}

Because of dense deployments and high molecular absorption losses, the LoS transmissions become dominant over NLoS transmissions. The channel power for the LoS communication is modeled between users and $\mathrm{THz}$ small cells as

$$
l(x)=\exp \left(-k\left(f_{c, 3}\right) x\right) x^{-\beta_{3}},
$$

where $x$ denotes the distance, $f_{c, 3}$ represents the $\mathrm{THz}$ frequency and $k\left(f_{c, 3}\right)$ is the molecular absorption coefficient dependent on frequency [33]. The directional transmitter and receiver antenna gains $G_{t}^{T}(\Phi)$ and $G_{r}^{T}(\Phi)$ are modeled as

$$
G_{y}^{T}= \begin{cases}G_{y}^{T(\max )}, & |\Phi| \leq s_{y} \\ G_{y}^{T(\min )}, & |\Phi|>s_{y}\end{cases}
$$

where $\mathrm{y} \in\{t, r\}, \Phi$ represents the beamwidth angle, $s_{y}$ is the main lobe beamwidth, $G_{y}^{T(\max )}$ and $G_{y}^{T(\min )}$ represents the main lobe and side lobe beamforming gains, respectively.

The SINR of a user in tier 3 can be computed as,

$$
\mathrm{SINR}_{\mathrm{T}}=\frac{\overbrace{\bar{P}_{3} \psi_{3}}^{\tilde{P}_{3}} G_{t}^{T(\max )}(\Phi) G_{r}^{T(\max )}(\Phi) l(x)}{\sigma_{3}^{2}+I_{3}}
$$

where $I_{3}=\sum_{i \in \phi_{T} / c_{o}} P_{3} G_{y}^{T} l\left(x_{i}\right)$ is the cumulative interference from other TSCs, $x_{i}$ represents the distance, $\bar{P}_{3}$ denotes the transmit power of the $\mathrm{THz} \mathrm{BS}, \psi_{3}$ is the bias factor and $\sigma_{3}^{2}$ is the thermal noise.

\section{D. mmWave UAV tier}

UAVs are distributed in the considered region of interest according to a PPP, $\phi_{U A V}$, with density $\lambda_{4} . \lambda_{4}$ is divided into two independent PPPs with densities $\overline{\lambda_{4}}$ and $\tilde{\lambda}_{4} \cdot \bar{\lambda}_{4}$ represents the proportion of UAVs operating on mmWave band denoted by $\alpha \times \lambda_{4}$ whereas $\widetilde{\lambda}_{4}$ represents the proportion of UAVs operating on sub- $6 \mathrm{GHz}$ band denoted by $(1-\alpha) \times \lambda_{4}$. Because of the blockage effect in the mmWave band, the UAV network can be further divided into two independent PPPs. One non-homogeneous PPP, $\phi_{L}$, represents the LoS mmWave UAVs and has a density of $\alpha \times \lambda_{4} \times \mathcal{P}_{L o S}(x)$. Similarly, other non-homogeneous PPP, $\phi_{N}$, represents the NLoS mmWave UAVs with density $\alpha \times \lambda_{4} \times\left(1-\mathcal{P}_{L o S}(x)\right)$. A geometric model 
given in [19] is used for the derivation of LoS probability $\left(\mathcal{P}_{L o S}\right)$ and is given as

$$
\mathcal{P}_{L o S}=\frac{1}{1+a_{u} \exp \left[-b_{u}\left(\Lambda_{U A V}-a_{u}\right)\right]},
$$

where $a_{u}$ and $b_{u}$ are environment dependent parameters and $\Lambda_{\mathrm{UAV}}$ is the elevation angle. $\Lambda_{\mathrm{UAV}}$ is given by

$$
\Lambda_{U A V}=\arctan \left(\frac{h_{t}}{\sqrt{r^{2}-h_{t}^{2}}}\right)
$$

where $h_{t}$ denotes the height of the UAV, $r$ represents the $3 \mathrm{D}$ distance and $\sqrt{r^{2}-h_{t}^{2}}$ represents the horizontal distance between UAV and a user. The NLoS probability is given by

$$
\mathcal{P}_{N L O S}=1-\mathcal{P}_{L o S},
$$

Because of the existence of two links, LoS and NLoS, between the user and the UAV due to blockages, two different path loss functions for LoS and NLoS links exist and are given as,

$$
P L(r)=\left\{\begin{array}{l}
P L^{L}(r)=C_{L} r^{-\beta_{L}} \\
P L^{N L}(r)=C_{N} r^{-\beta_{N}}
\end{array},\right.
$$

where $C_{L}$ and $C_{N}$ are the intercepts for the LoS and NLoS formulas, $\beta_{L}$ and $\beta_{N}$ are the LoS and NLoS path loss exponents. The values of $\beta_{L}, \beta_{N}, C_{L}$ and $C_{N}$ are found using field tests [13], [34]. The antenna gain for the UAV mmWave network, $G_{e}^{u a v}$, where $e \in\{1,2,3,4\}$ is given as,

$$
G_{e}^{u a v}= \begin{cases}G_{1}=G_{r}^{u a v} G_{t}^{\text {uav }}, & \text { with prob. } \frac{\Theta_{r} \Theta_{\text {uav }}}{4 \pi^{2}} \\ G_{2}=G_{r}^{u a v} g_{t}^{u a v}, & \text { with prob. } \frac{\Theta_{r}\left(2 \pi-\Theta_{\text {uav }}\right)}{4 \pi^{2}} \\ G_{3}=g_{r}^{\text {uav }} G_{t}^{\text {uav }}, & \text { with prob. } \frac{\left(2 \pi-\Theta_{r}\right) \Theta_{\text {uav }}}{4 \pi^{2}} \\ G_{4}=g_{r}^{\text {uav }} g_{t}^{\text {uav }}, & \text { with prob. } \frac{\left(2 \pi-\Theta_{r}\right)\left(2 \pi-\Theta_{\text {uav }}\right)}{4 \pi^{2}}\end{cases}
$$

where $G_{t}^{u a v}, G_{r}^{u a v}$ and $g_{t}^{u a v}, g_{r}^{u a v}$ are main lobe gains and side lobe gains for transmitter and receiver, and the half power beamwidths of transmitter and receiver are given by $\Theta_{\text {uav }}$ and $\Theta_{r}$.

Considering now the user associates with LoS and NLoS UAV $u_{o}$ at a distance $r$, then the SINR can be given as,

$$
\mathrm{SINR}_{U A V}=\frac{\overbrace{\bar{P}_{4} \psi_{4}}^{\tilde{P}_{4}} r^{-} \beta_{4} h_{r} G_{r}^{\text {uav }} G_{t}^{\text {uav }}}{I_{4}+\sigma_{4}^{2}}
$$

where $\bar{P}_{4}$ is the UAV transmit power, $h$ is the Nakagami fading, $G_{e}^{u a v}$ is the antenna gain, $\psi_{4}$ is the bias factor, $I_{4}=\sum_{i \in(l, n)} \sum_{j \in\left\{\phi_{U A V} / u_{o} \cup \phi_{m m}\right\}} P_{4} G_{e}^{u a v} h_{j} r_{j}^{-\beta_{4 i}}$ is the aggregate interference power from other UAVS and mmWave small cells, and $\sigma_{4}^{2}$ is the noise power spectral density.

\section{Performance Evaluation}

In this section, we derive the association and coverage probabilities of a typical user, assumed at origin, which is connected to one of the HetNet tiers. We start with the following lemma.
Lemma 1 . The association probability that a typical user connects with the $i^{\text {th }}$ tier based on maximum biased received power is given as

$$
\begin{aligned}
& \delta_{i}=\mathbb{E}_{x_{i}}\left[\mathbb{P}\left[P_{r, i}>\max _{n, n \neq i} P_{r, n}\right]\right] \\
& =\mathbb{E}_{x_{i}}\left[\prod_{n=1, n \neq i}^{4}\left[\mathbb{P}\left[P_{r, i}>P_{r, n}\right]\right]\right. \\
& \stackrel{(a)}{=} \mathbb{E}_{x_{i}}\left[\prod_{n=1, n \neq i}^{4}\left[\mathbb{P}\left[x_{n}>\left(\frac{\widetilde{P}_{n} G_{n} x_{i}^{\beta_{i}}}{\widetilde{P}_{i} G_{i}}\right)^{1 / \beta_{n}}\right]\right]\right] \\
& =\int_{0}^{\infty}[\underbrace{\prod_{n=1, n \neq i}^{4}\left[\mathbb{P}\left[x_{n}>\left(\frac{\widetilde{P}_{n} G_{n} x_{i}^{\beta_{i}}}{\widetilde{P}_{i} G_{i}}\right)^{1 / \beta_{n}}\right]\right]}_{\kappa}] f_{X_{i}}(x) d x,
\end{aligned}
$$

where (a) is based on downlink user association. The detailed derivation of Lemma 1 is given in Appendix A.

From Lemma 1, we can infer that the typical association of a user to a particular tier depends upon the distance between the user and the BS, transmit power, path loss exponent and antenna gains of that tier. The user will associate to the BS that provides the maximum biased received power and the association probability to a particular tier can be increased by increasing the transmit power or the bias of that particular tier. This biasness helps in offloading the users to tiers with high available bandwidth.

Now that the user association probability with a particular tier is known, we now revert our attention on finding the coverage probability of a user with that particular tier. Please refer to the following lemma.

Lemma 2 . The SINR coverage probability at the user connecting with MBS is given by

$$
P_{c}^{1}\left(\Gamma_{1}\right)=\int_{0}^{\infty} P_{c}^{1}\left(\Gamma_{1}, x\right) f_{X_{1}}(x) d x
$$

where $P_{c}^{1}\left(\Gamma_{1}, x\right)$ denotes the conditional coverage probability for a typical user and the serving MBS for a distance $x$ and $f_{X_{1}}(x)$ is the PDF of the distance between the typical user and $M B S$ and is given as

$$
\begin{aligned}
f_{X_{1}}(x) & =\frac{2 \pi \lambda_{1}}{\delta_{1}} \operatorname{xexp}\left(-\pi \lambda_{1} x^{2}-2 \pi \lambda_{2} \Upsilon(x)\right. \\
& \left.-2 \pi \lambda_{3} T(x)-2 \pi \lambda_{4} U(x)\right),
\end{aligned}
$$

where $\delta_{1}$ is the user association probability with tier 1 and is given as,

$$
\begin{aligned}
\delta_{1} & =2 \pi \lambda_{1} \int_{0}^{\infty} \operatorname{xexp}\left(-\pi \lambda_{1} x^{2}-2 \pi \lambda_{2} \Upsilon(x)-2 \pi \lambda_{3} T(x)\right. \\
& \left.-2 \pi \lambda_{4} U(x)\right) d x
\end{aligned}
$$

The conditional coverage probability $P_{c}^{1}\left(\Gamma_{1}, x\right)$, is given as,

$$
P_{c}^{1}\left(\Gamma_{1}, x\right)=\exp \left(\frac{-\Gamma_{1} x^{\beta_{1}} \sigma_{1}^{2}}{\widetilde{P}_{1}}\right) \mathbb{E}_{I_{1}}\left[\mathcal{L}_{I_{1}}\left(\frac{\Gamma_{1} x^{\beta_{1}}}{\widetilde{P}_{1}}\right)\right]
$$


Proof. See Appendix B.

Lemma 3 . The SINR coverage probability of a user connecting with MSC tier is given as,

$$
P_{c}^{2}\left(\Gamma_{2}\right)=\sum_{v \in\{L, N\}} \delta_{2, v} P_{c}^{2, v}\left(\Gamma_{2}\right),
$$

where $P_{c}^{2, L}\left(\Gamma_{2}\right)$ and $P_{c}^{2, N}\left(\Gamma_{2}\right)$ are the conditional coverage probabilities that a user is connected with MSC in $\phi_{L}$ and $\phi_{N}$, respectively. $P_{c}^{2, v}\left(\Gamma_{2}\right)$ can be computed as,

$$
\begin{aligned}
P_{c}^{2, v}\left(\Gamma_{2}\right) & =\sum_{i=1}^{\Omega_{w}}(-1)^{i+1}\left(\begin{array}{c}
\Omega_{w} \\
i
\end{array}\right) \int_{0}^{\infty} \exp \left(\frac{-i \rho_{w} x^{\beta_{v}} \Gamma_{2} \sigma_{2}^{2}}{G_{r}^{m m} G_{t}^{m m}}\right. \\
& \left.-E_{v, i}\left(\Gamma_{2}, x\right)-H_{v, i}\left(\Gamma_{2}, r\right)\right) f_{v}(x) d x,
\end{aligned}
$$

where $E_{v, i}\left(\Gamma_{2}, x\right)$ and $H_{v, i}\left(\Gamma_{2}, r\right)$ accounts for the interference terms from LoS(NLoS) mmWave small cells and UAVs, $\rho_{w}=$ $\Omega_{w}\left(\Omega_{w} !\right)^{-1 / \Omega_{w}}, w \in\left\{l_{m}, n_{m}\right\}, F\left(\Omega_{w}, x\right)=1-\frac{1}{(1+x)^{\Omega_{w}}}$ and $\hat{a}_{i}=\frac{a_{i}}{G_{r}^{m m} G_{t}^{m m}}$.

$$
\begin{gathered}
E_{L, i}\left(\Gamma_{2}, x\right)=2 \pi \lambda_{2} \sum_{i=1}^{4} p_{i}\left[\int_{x}^{\infty} F\left(\Omega_{l_{m}}, \frac{i \rho_{l_{m}} \hat{a}_{i} \Gamma_{2} x^{\beta_{L}}}{\Omega_{l_{m}} \beta_{L}}\right)\right. \\
t \mathfrak{P}_{L o S}(t) d t+\int_{\Xi_{L}(x)}^{\infty} F\left(\Omega_{n_{m}}, \frac{i \rho_{l_{m}} \hat{a}_{i} \Gamma_{2} x^{\beta_{L}}}{\Omega_{n_{m}} \beta_{N}}\right) \\
\left.t\left(1-\mathfrak{P}_{L o S}(t)\right) d t\right]
\end{gathered}
$$$$
E_{N, i}\left(\Gamma_{2}, x\right)=2 \pi \lambda_{2} \sum_{i=1}^{4} p_{i}\left[\int_{\Xi_{N}(x)}^{\infty} F\left(\Omega_{l_{m}}, \frac{i \rho_{n_{m}} \hat{a}_{i} \Gamma_{2} x^{\beta_{N}}}{\Omega_{l_{m}} \beta_{L}}\right)\right.
$$$$
t \mathfrak{P}_{L o S}(t) d t+\int_{x}^{\infty} F\left(\Omega_{n_{m}}, \frac{i \rho_{n_{m}} \hat{a}_{i} \Gamma_{2} x^{\beta_{N}}}{\Omega_{n_{m}} t \beta_{N}}\right)
$$$$
\left.t\left(1-\mathfrak{P}_{L o S}(t)\right) d t\right],
$$

$$
\begin{gathered}
H_{L, i}\left(\Gamma_{2}, r\right)=2 \pi \alpha \lambda_{4} \sum_{i=1}^{4} p_{i}\left[\int_{r}^{\infty} F\left(\Omega_{l_{m}}, \frac{i \rho_{l, m} \hat{a}_{i} \Gamma_{2} x^{\beta_{L}}}{\Omega_{l_{m} t} t_{L}}\right)\right. \\
t \mathcal{P}_{L o S}(t) d t+\int_{\xi_{L}(r)}^{\infty} F\left(\Omega_{n_{m}}, \frac{i \rho_{l, m} \hat{a}_{i} \Gamma_{2} x^{\beta_{L}}}{\Omega_{n_{m}} \beta}\right) \\
\left.t\left(1-\mathcal{P}_{L o S}(t)\right) d t\right]
\end{gathered}
$$$$
H_{N, i}\left(\Gamma_{2}, r\right)=2 \pi \alpha \lambda_{4} \sum_{i=1}^{4} p_{i}\left[\int_{\xi_{N}(r)}^{\infty} F\left(\Omega_{l_{m}}, \frac{i \rho_{n_{m}} \hat{a}_{i} \Gamma_{2} x^{\beta_{N}}}{\Omega_{l_{m}} t_{L}}\right)\right.
$$$$
t \mathcal{P}_{L o S}(t) d t+\int_{r}^{\infty} F\left(\Omega_{n_{m}}, \frac{i \rho_{n_{m}} \hat{a}_{i} \Gamma_{2} x^{\beta_{N}}}{\Omega_{n_{m}} t^{\beta_{N}}}\right)
$$$$
\left.t\left(1-\mathcal{P}_{L o S}(t)\right) d t\right],
$$

Proof. See Appendix C.

Lemma 4 . The SINR coverage probability of a user connecting with TSC tier is given as,

$$
P_{c}^{3}\left(\Gamma_{3}\right)=\sum_{v \in\{L, N\}} \delta_{3, v} P_{c}^{3, v}\left(\Gamma_{3}\right),
$$

The conditional probability $P_{c}^{3, v}\left(\Gamma_{3}\right)$ can be derived as

$$
\begin{aligned}
P_{c}^{3, v}\left(\Gamma_{3}\right) & =\sum_{i=1}^{\Omega}(-1)^{i+1}\left(\begin{array}{c}
\Omega_{w} \\
i
\end{array}\right) \int_{0}^{\infty} \exp \left(\frac{-i \rho_{w} x^{\beta_{v}} \Gamma_{3} \sigma_{3}^{2}}{G_{r}^{T} G_{t}^{T}}\right. \\
& \left.-S_{v, i}\left(\Gamma_{3}, x\right)\right) f_{X_{3}}(x) d x,
\end{aligned}
$$

where $f_{X_{3}}(x)$ is the PDF of the distance between the typical user and TSC tier. $S_{v, i}\left(\Gamma_{3}, x\right)$ in (29) can be computed using similar steps followed in Appendix $C$.

Proof. Proof follows similar steps introduced in Appendix C.

Lemma 5 . The SINR coverage probability of a user connecting with $U A V$ tier is given as,

$$
P_{c}^{4}\left(\Gamma_{4}\right)=\sum_{v \in\{L, N\}} \delta_{4, v} P_{c}^{4, v}\left(\Gamma_{4}\right),
$$

where $P_{c}^{4, L}\left(\Gamma_{4}\right)$ and $P_{c}^{4, N}\left(\Gamma_{4}\right)$ are the conditional coverage probabilities that a user is connected with UAV tier in $\phi_{L}$ and $\phi_{N}$, respectively. $P_{c}^{4, v}\left(\Gamma_{4}\right)$ can be computed as

$$
\begin{aligned}
P_{c}^{4, v}\left(\Gamma_{4}\right) & =\sum_{i=1}^{\Omega_{w}}(-1)^{i+1}\left(\begin{array}{c}
\Omega_{w} \\
i
\end{array}\right) \int_{h_{t}}^{\infty} \exp \left(\frac{-i \rho_{w} r^{\beta_{v}} \Gamma_{4} \sigma_{4}^{2}}{G_{r}^{u a v} G_{t}^{u a v}}\right. \\
& -H_{v, i}\left(\Gamma_{4}, r\right)-E_{v, i}\left(\Gamma_{4}, x\right) f_{v}(r) d r,
\end{aligned}
$$

where $f_{v}(r)$ is the PDF of the distance between a user and UAV tier, $\rho_{w}=\Omega_{w}\left(\Omega_{w} !\right)^{-1 / \Omega_{w}}, w \in\left\{l_{m}, n_{m}\right\}, H_{v, i}\left(\Gamma_{4}, r\right)$ and $E_{v, i}\left(\Gamma_{4}, r\right)$ accounts for the interference terms from LoS(NLoS) mmWave UAVs and mmWave small cells, where

$$
\begin{gathered}
H_{L, i}\left(\Gamma_{4}, r\right)=2 \pi \alpha \lambda_{4} \sum_{i=1}^{4} p_{i}\left[\int_{r}^{\infty} F\left(\Omega_{l_{m}}, \frac{i \rho_{l_{m}} \hat{a}_{i} \Gamma_{4} r^{\beta_{L}}}{\Omega_{l_{m}} \beta^{\beta_{L}}}\right)\right. \\
t \mathcal{P}_{L o S}(t) d t+\int_{\xi_{L}(r)}^{\infty} F\left(\Omega_{n_{m}}, \frac{i \rho_{l_{m}} \hat{a}_{i} \Gamma_{4} r^{\beta_{L}}}{\Omega_{n_{m}} t^{\beta_{N}}}\right) \\
\left.t\left(1-\mathcal{P}_{\text {LoS }}(t)\right) d t\right], \\
H_{N, i}\left(\Gamma_{4}, r\right)=2 \pi \alpha \lambda_{4} \sum_{i=1}^{4} p_{i}\left[\int_{\xi_{N}(r)}^{\infty} F\left(\Omega_{l_{m}}, \frac{i \rho_{n_{m}} \hat{a}_{i} \Gamma_{4} r^{\beta_{N}}}{\Omega_{l_{m}} t \beta_{L}}\right)\right. \\
t \mathcal{P}_{\text {LoS }}(t) d t+\int_{r}^{\infty} F\left(\Omega_{n_{m}}, \frac{i \rho_{n_{m}} \hat{a}_{i} \Gamma_{4} r^{\beta_{N}}}{\Omega_{n_{m}} t \beta_{N}}\right) \\
\left.t\left(1-\mathcal{P}_{L o S}(t)\right) d t\right],
\end{gathered}
$$


TABLE I: Simulation Parameters

$$
\begin{gathered}
E_{L, i}\left(\Gamma_{4}, x\right)=2 \pi \lambda_{2} \sum_{i=1}^{4} p_{i}\left[\int_{x}^{\infty} F\left(\Omega_{l_{m}}, \frac{i \rho_{l_{m}} \hat{a}_{i} \Gamma_{4} x^{\beta_{L}}}{\Omega_{l_{m}} \beta^{\beta_{L}}}\right)\right. \\
t \mathfrak{P}_{L o S}(t) d t+\int_{\Xi_{L}(x)}^{\infty} F\left(\Omega_{n_{m}}, \frac{i \rho_{l_{m}} \hat{a}_{i} \Gamma_{4} \beta^{\beta_{L}}}{\Omega_{n_{m}} t \beta_{N}}\right) \\
\left.t\left(1-\mathfrak{P}_{\text {LoS }}(t)\right) d t\right] \\
E_{N, i}\left(\Gamma_{4}, x\right)=2 \pi \lambda_{2} \sum_{i=1}^{4} p_{i}\left[\int_{\Xi_{N}(x)}^{\infty} F\left(\Omega_{l_{m}}, \frac{i \rho_{n_{m}} \hat{a}_{i} \Gamma_{4} x^{\beta_{N}}}{\Omega_{l_{m}} t \beta_{L}}\right)\right. \\
t \mathfrak{P}_{L o S}(t) d t+\int_{x}^{\infty} F\left(\Omega_{n_{m}}, \frac{i \rho_{n_{m}} \hat{a}_{i} \Gamma_{4} x^{\beta_{N}}}{\Omega_{n_{m}} t \beta_{N}}\right) \\
\left.t\left(1-\mathfrak{P}_{\text {LoS }}(t)\right) d t\right]
\end{gathered}
$$

Proof. The proof is omitted due to the space limitations and can be derived on the similar lines following Appendix C.

Special Case: By setting $\Omega_{l_{m}}=\Omega_{n_{m}}=\rho_{l_{m}}=\rho_{n_{m}}=1$, and taking the density for LoS sub-6 GHz UAVs as $(1-\alpha) \times \lambda_{4} \times$ $\mathcal{P}_{\text {LoS }}(x)$ and for NLoS sub-6 GHz UAVs as $(1-\alpha) \times \lambda_{4} \times$ $\left(1-\mathcal{P}_{\operatorname{LoS}}(x)\right)$, Eq. (31) can be transformed into conditional coverage probability for sub- $6 \mathrm{GHz}$ UAVs. The conditional coverage probability for sub- $6 \mathrm{GHz}$ UAVs is then given as,

$$
P_{c}^{4, v}\left(\Gamma_{4}\right)=\int_{h_{t}}^{\infty} \exp \left(\frac{-i r^{\beta_{v}} \Gamma_{4} \sigma_{4}^{2}}{G_{r}^{u a v} G_{t}^{u a v}}-H_{v, i}\left(\Gamma_{4}, r\right)\right) f_{v}(r) d r,
$$

where $H_{v, i}\left(\Gamma_{4}, r\right)$ are the interferences from the LoS and NLoS sub-6 GHz UAVs. The Proof is omitted due to the space limitations and can be computed following similar steps used in Appendix C.

From the above mentioned lemmas, we can see that the SINR coverage probability of a user connecting to a particular tier depends upon the SINR threshold. As we increase the threshold value, SINR coverage probability tends to decrease as less number of users remains in the coverage.

Proposition 1 . The total coverage probability, $P_{c, T}$, for the multi-tier hybrid network is defined as

$$
P_{c, T}=\sum_{i=1}^{4} P_{c}^{i} \delta_{i}
$$

where $\delta_{i}$ represents the user association probability for tier $i$ and $P_{c}^{i}$ denotes the coverage probability for tier $i$.

Lemma 6 . The achievable ergodic rate for a user connecting with $i^{\text {th }}$ tier is given as,

$$
R_{i}=\frac{1}{\ln 2} \int_{0}^{\infty} \frac{P_{c}^{i}\left(\Gamma_{i}\right)}{1+\Gamma_{i}} d \Gamma_{i},
$$

where $P_{c}^{i}$ is the coverage probability of tier $i$ and $\Gamma_{i}$ is the SINR threshold of tier $i$.

Proof. See Appendix D.

\begin{tabular}{|c|c|c|c|}
\hline Parameter & Value & Parameter & Value \\
\hline$f_{c, 2}, f_{c, 4}$ & $28 \mathrm{GHz}$ & $B_{M S C}, B_{U A V}$ & $100 \mathrm{MHz}$ \\
\hline$f_{c, 1}$ & $2.4 \mathrm{GHz}$ & $B_{M B S}, B_{T S C}$ & $20 \mathrm{MHz}, 1 \mathrm{GHz}$ \\
\hline$f_{c, 3}$ & $1 \mathrm{THz}$ & $f_{c}($ sub-6 GHz $U A V s)$ & $2.6 \mathrm{GHz}$ \\
\hline$g_{r}^{\text {mm }}, g_{r}^{\text {uav }}, g_{r}^{T}$ & $-10 \mathrm{~dB}$ & $g_{t}^{\text {mm }}, g_{t}^{\text {uav }}, g_{t}^{T}$ & $0 \mathrm{~dB}$ \\
\hline$\beta_{1}$ & 4 & $\beta_{L}, \beta_{N}$ & 2,4 \\
\hline$\overline{P_{1}}$ & $40 \mathrm{~W}$ & $\overline{P_{2}}, \overline{P_{3}}, \overline{P_{4}}$ & $1 \mathrm{~W}$ \\
\hline$G_{r}^{T}, G_{t}^{T}$ & $25 \mathrm{~dB}$ & $G_{r}^{m m}, G_{t}^{m m}, G_{r}^{\text {uav }}, G_{r}^{\text {uav }}$ & $10 \mathrm{~dB}$ \\
\hline$a_{u}, b_{u}$ & $9,0.11$ & $k(f)$ & $.05 m^{-1}$ \\
\hline $\mathrm{h}_{t}$ & $50 \mathrm{~m}$ & $\theta_{r}, \theta_{t}$ & $90^{\circ}, 30^{\circ}$ \\
\hline
\end{tabular}

From Lemma 6, rate coverage probability can be defined for a given threshold, $\tau$, as

$$
P_{\text {rate }, i}(\tau)=P\left(R_{i}>\tau\right),
$$

where $\tau$ is the rate coverage probability threshold and is assumed to be same for all tiers.

From Lemma 6, we can see that rate coverage probability depends upon the rate threshold. A higher threshold value implies that less number of users will satisfy the higher data rate requirements. The higher data rate requirements can be met if the users are connected to mmWave or $\mathrm{THz}$ frequency tiers because of the higher available bandwidth.

Proposition 2 . The total rate coverage probability, $P_{\text {rate }}$, for the HetNet is defined as

$$
P_{\text {rate }}(\tau)=\sum_{i=1}^{4} P_{\text {rate }, i} \delta_{i},
$$

where $\delta_{i}$ is the association probability of tier $i$ and $P_{\text {rate }, i}$ is the rate coverage probability of the tier $i$.

\section{Performance Evaluation and Discussion}

In this section, we present the simulation results for the proposed hybrid HetNet. In our simulation setup, we assume the MBS density to be $\lambda_{1}=\frac{3}{500^{2} \times \pi}$. The downlink transmission powers are assumed to be 40 Watts for MBS and 1 Watt for other tiers. The transmission frequency $f_{c, 1}$ is set to be 2.4 $\mathrm{GHz}, f_{c, 3}$ is taken as $1 \mathrm{THz}, f_{c, 2}$ and $f_{c, 4}$ are taken as 28 GHz. Furthermore, Table I lists the detailed parameters used for simulation setup. The results are averaged over $10^{6}$ Monte Carlo iterations.

Fig. 2 depicts the probability of user association to each tier versus different TSC densities. It can be seen in Fig. 2, that if we increase the TSC density, user association probability increases as more users start to associate with the TSC. In Fig. 2 by increasing the ratio of densities from 5 to 25 , the user association probability increases by $32 \%$ from 0.2 to 0.52 . This offloading of users from other tiers to TSC, results in a decrease in the association probabilities of other tiers. For the same increase in density ratio, $18 \%$ of users offloaded from mmWave tier. So there is a tradeoff between having better SINR coverage at lower frequencies than having a better rate coverage at higher frequencies. This increased association to TSC helps to fulfill the users demand of very high data rates. It can also be seen in the Fig. 2 that analytical results are in compliance with the simulation results. 


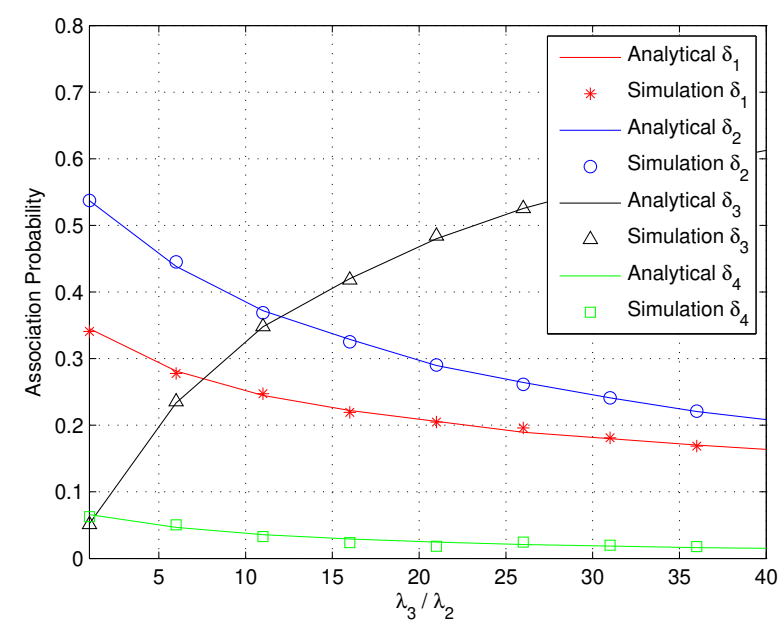

Fig. 2: Association probability versus varying $\lambda_{3}$ with $\lambda_{1}=$ $4 \times 10^{-6} \mathrm{BS} / \mathrm{m}^{2}, \lambda_{2}=3 \times \lambda_{1}, \mathrm{BS} / \mathrm{m}^{2}, \lambda_{4}=3 \times \lambda_{1} \mathrm{BS} / \mathrm{m}^{2}$ and $\psi_{1}=\psi_{2}=0 \mathrm{~dB}, \psi_{3}=\psi_{4}=5 \mathrm{~dB}$.

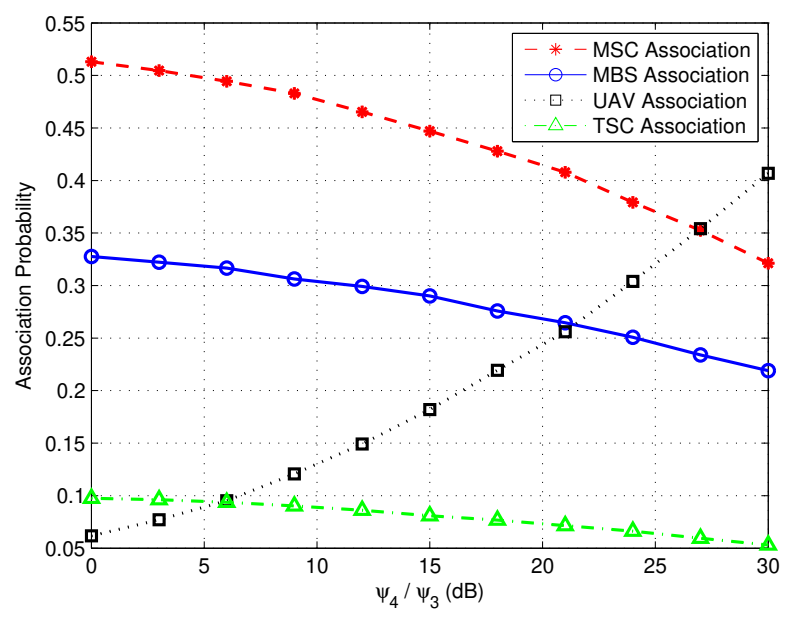

Fig. 3: User association probability vs. varying $\psi_{4}$ with $\lambda_{1}=4 \times 10^{-6} \mathrm{BS} / \mathrm{m}^{2}, \lambda_{2}=3 \times \lambda_{1} \mathrm{BS} / \mathrm{m}^{2}, \lambda_{3}=2 \times \lambda_{2} \mathrm{BS} / \mathrm{m}^{2}$, $\lambda_{4}=3 \times \lambda_{1} \mathrm{BS} / \mathrm{m}^{2}, \psi_{3}=5 \mathrm{~dB}, \psi_{1}=\psi_{2}=0 \mathrm{~dB}$.

Fig. 3 demonstrates the user association probabilities versus ratio of bias values for UAV tier w.r.t. TSC tier. This biasness is necessary in order to overcome the high propagation losses encountered by UAVs at mmWave frequencies and also assists in offloading the users from the sub- $6 \mathrm{GHz}$ BSs to mmWave UAVs. In Fig. 3, the $\psi_{3}$ is fixed to be $5 \mathrm{~dB}$. We can see an increasing trend in the user association probability with UAV tier for an increased $\psi_{4}$ w.r.t. $\psi_{3}$. For example, if we increase the ratio from $0 \mathrm{~dB}$ to $30 \mathrm{~dB}$, there is an increase of about $35 \%$ in user association with UAV. This increased association of users with UAV results in a lower association of users with other tiers. For the same increase in ratio, we can see a decline in user association with MSC, MBS and TSC by $20 \%, 11 \%$, and $5 \%$, respectively.

Fig. 4 depicts the SINR coverage probability of HetNet versus ratio of bias values for UAV tier w.r.t. TSC tier for

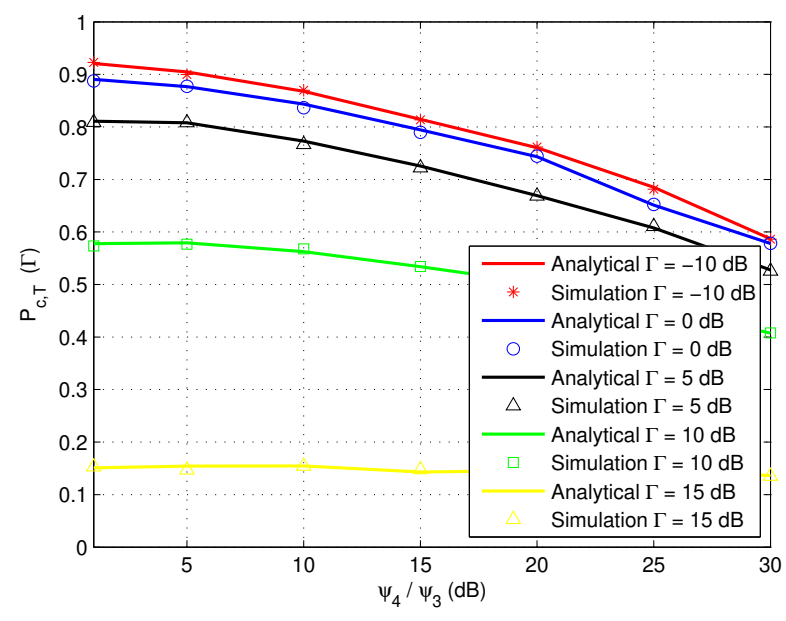

Fig. 4: SINR coverage probability versus varying $\psi_{4}$ with $\lambda_{1}=$ $4 \times 10^{-6} \mathrm{BS} / \mathrm{m}^{2}, \lambda_{2}=10 \times \lambda_{1} \mathrm{BS} / \mathrm{m}^{2}, \lambda_{3}=3 \times \lambda_{2} \mathrm{BS} / \mathrm{m}^{2}$, $\lambda_{4}=10 \times \lambda_{1} \mathrm{BS} / \mathrm{m}^{2}, \psi_{1}=\psi_{2}=0 \mathrm{~dB}, \psi_{3}=30 \mathrm{~dB}$ and $\Gamma_{1}=$ $\Gamma_{2}=\Gamma_{3}=\Gamma_{4}=\Gamma=0 \mathrm{~dB}$.

various SINR threshold values. It can be seen in Fig. 4 that with an increase in biasness, more users start to associate with mmWave UAVs. This results in a decrease in SINR coverage probability of HetNet because of the high propagation losses experienced by users at mmWave frequencies. In Fig. 4, it can be seen that for an increase of ratio from $0 \mathrm{~dB}$ to 30 $\mathrm{dB}$ for an SINR threshold of $-10 \mathrm{~dB}$, the number of users in coverage reduces by $34 \%$ from 0.92 to 0.58 . This figure also shows the impact of various SINR thresholds on coverage probability of HetNet. In Fig. 4, we can see that by increasing the SINR threshold from $-10 \mathrm{~dB}$ to $15 \mathrm{~dB}$, the number of users in coverage reduces from $85 \%$ to $15 \%$ for a biased ratio of 10 dB.

Fig. 5 depicts the UAV association probability and SINR coverage probability of HetNet versus transmit power of UAVs. It can be seen in Fig. 5 that when the transmit power is increased from 1 Watts to 10 Watts more user starts to associate with UAVs. As UAVs are operating on mmWave frequencies, any user associated with UAV will face high propagation losses as opposed to the users associated with sub$6 \mathrm{GHz}$ frequency band. This increased association of users with mmWave UAVs results in a decreased SINR coverage probability of HetNet. If we increase the transmit power of UAVs from 1 Watts to 10 Watts, we can a see $13 \%$ decline in the number of users in SINR coverage. In Fig. 5 we also seen an increase in user association with an increase in transmit power. This increase means more users will now experience high data rates available at mmWave frequencies.

Fig. 6 shows the variation in rate coverage probability versus different rate threshold values $\tau$ for different densities of $\mathrm{THz}$ cells. The values of $\tau$ has been taken from $1 \mathrm{Mbps}$ to $1 \mathrm{Gbps}$. It is shown that if we increase the rate thresholds lesser number of users remains in the coverage. In Fig. 6 we can see that for the same density of MSC BSs and TSC BSs, around $75 \%$ of the users are getting data rates around $100 \mathrm{Mbps}$ and $12 \%$ of the users receive data rates up to 1 Gbps. Fig. 6 also depicts 


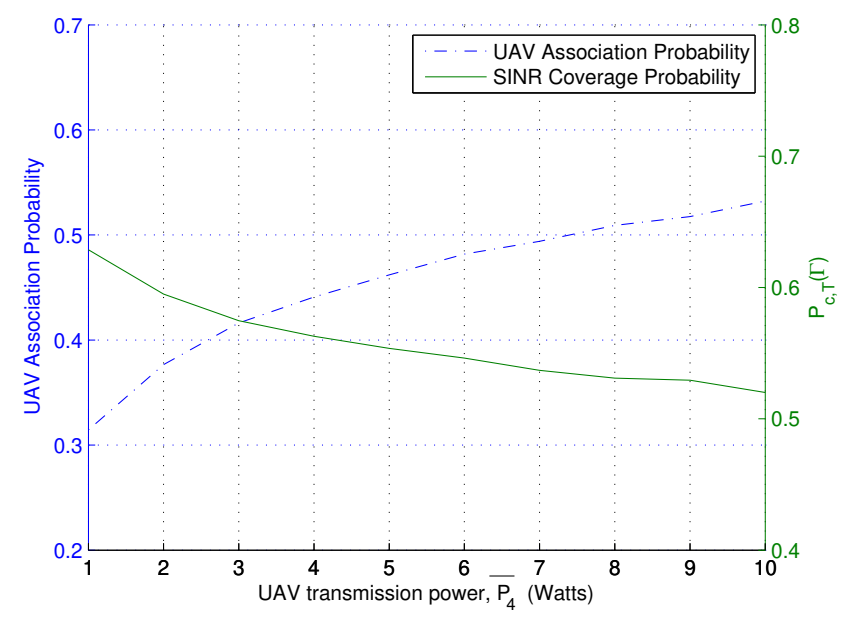

Fig. 5: Impact of UAV transmit power on user association with UAVs and SINR coverage probability with $\lambda_{1}=$ $4 \times 10^{-6} \mathrm{BS} / \mathrm{m}^{2}, \lambda_{2}=10 \times \lambda_{1} \mathrm{BS} / \mathrm{m}^{2}, \lambda_{3}=3 \times \lambda_{2} \mathrm{BS} / \mathrm{m}^{2}$, $\lambda_{4}=10 \times \lambda_{1} \mathrm{BS} / \mathrm{m}^{2}, \psi_{1}=\psi_{2}=0 \mathrm{~dB}, \psi_{3}=30 \mathrm{~dB}, \psi_{4}=10$ $\mathrm{dB}$ and $\Gamma_{1}=\Gamma_{2}=\Gamma_{3}=\Gamma_{4}=\Gamma=0 \mathrm{~dB}$.

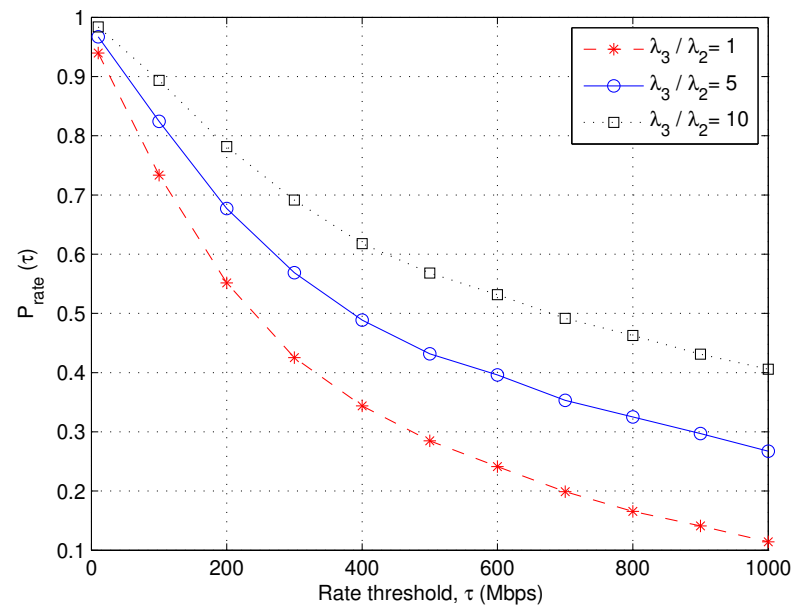

Fig. 6: Rate coverage probability versus rate thresholds $\tau$ for different $\tau$ with $\lambda_{1}=4 \times 10^{-6} \mathrm{BS} / \mathrm{m}^{2}, \lambda_{2}=2 \times \lambda_{1} \mathrm{BS} / \mathrm{m}^{2}$, $\lambda_{4}=2 \times \lambda_{1} \mathrm{BS} / m^{2}, \psi_{1}=\psi_{2}=0 \mathrm{~dB}, \psi_{3}=20 \mathrm{~dB}, \psi_{4}=20$ $\mathrm{dB}$.

the effects of increasing the TSC density on the achievable data rates. An increase in TSC density means more users are now associated with $\mathrm{THz}$ small cells which results in higher achievable data rates. In Fig. 6, it is shown that if we increase the TSC density from 1 to 10,90\% of the users are getting the data rates up to $100 \mathrm{Mbps}$.

Fig. 7 depicts the variations in rate coverage probability curves versus rate thresholds for various TSC bandwidth settings. The density of the THz BSs is assumed to be fixed. From Fig. 7 we can see a similar decline in rate coverage probability as we observed in Fig. 6. As we increase the rate threshold, less number of users are able to satisfy higher threshold values which results in decreased coverage probability. In Fig. 7, it

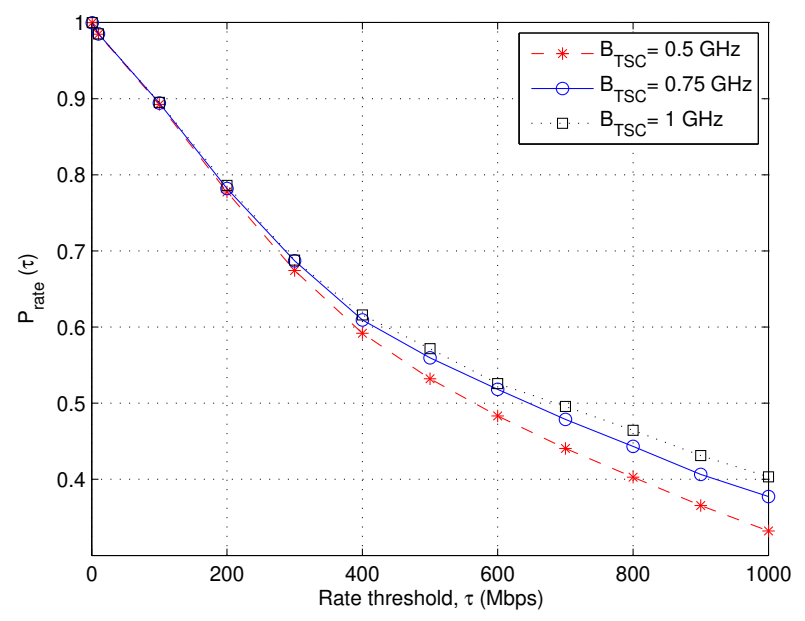

Fig. 7: Rate coverage probability versus rate thresholds $\tau$ for various $\mathrm{THz}$ Bandwidths with $\lambda_{1}=4 \times 10^{-6} \mathrm{BS} / \mathrm{m}^{2}, \lambda_{2}=$ $2 \times \lambda_{1} \mathrm{BS} / \mathrm{m}^{2}, \lambda_{3}=5 \times \lambda_{2} \mathrm{BS} / \mathrm{m}^{2}, \lambda_{4}=2 \times \lambda_{1} \mathrm{BS} / \mathrm{m}^{2}, \psi_{1}=$ $\psi_{2}=0 \mathrm{~dB}, \psi_{3}=20 \mathrm{~dB}, \psi_{4}=20 \mathrm{~dB}$.

is shown that if we increase the threshold from $200 \mathrm{Mbps}$ to $800 \mathrm{Mbps}$ for $0.5 \mathrm{GHz}$ TSC bandwidth, the rate coverage probability decreases by $38 \%$. Fig. 7 also depicts the effect of an increased bandwidth on coverage probability at $\mathrm{THz}$ frequencies. In Fig. 7 we can see that for a fixed TSC density, if we increase the bandwidth from $0.5 \mathrm{GHz}$ to $1 \mathrm{GHz}$ more number of users start to experience higher data rates. For a rate threshold of $600 \mathrm{Mbps}$, if we increase the bandwidth of TSC from $0.5 \mathrm{GHz}$ to $1 \mathrm{GHz}, 4 \%$ more users will come into coverage. An increase in bandwidth allows more users to experience high data rates as the capacity increases with an increase in available bandwidth.

Fig. 8a depicts the SINR coverage probability versus proportion of sub- $6 \mathrm{GHz}$ UAVs to mmWave-enabled UAVs in the HetNet for various SINR threshold values. In Fig. 8a, $(1-\alpha)$ represents the proportion of UAVs operating on sub- $6 \mathrm{GHz}$ frequency in the HetNet. Sub-6 GHz UAVs are assumed to operate at $2.6 \mathrm{GHz}$ frequency. It is also assumed that the sub$6 \mathrm{GHz}$ UAVs use directional antennas with antenna gain of 10 dB. From Fig. 8a we can see that if we increase the proportion of sub- $6 \mathrm{GHz}$ UAVS by $0 \%$ to $100 \%$ by varying the value of tuning parameter $1-\alpha$ from 0 (all mmWave UAVs) to 1 (all sub-6 GHz UAVs), there is an increase in the SINR coverage probability. This increase is due to fact that there are less propagation losses at sub- $6 \mathrm{GHz}$ frequencies than the mmWave frequencies. In Fig. 8a, for an SINR threshold of $10 \mathrm{~dB}$, an increase of $16 \%$ in SINR coverage probability is observed when sub-6 GHz UAVs are increased from $0 \%$ to $100 \%$ in the HetNet. Fig. 8a also shows the effect of increasing SINR threshold from $-10 \mathrm{~dB}$ to $10 \mathrm{~dB}$ on SINR coverage probability. It can be seen from the Fig. 8a that an increase in the SINR threshold results in less number of users being in coverage. For example, if we have $60 \%$ sub- $6 \mathrm{GHz}$ UAVs in the HetNet, $90 \%$ of users are in coverage for an SINR threshold of $0 \mathrm{~dB}$. If we increase the SINR threshold for the same proportion of sub-6 


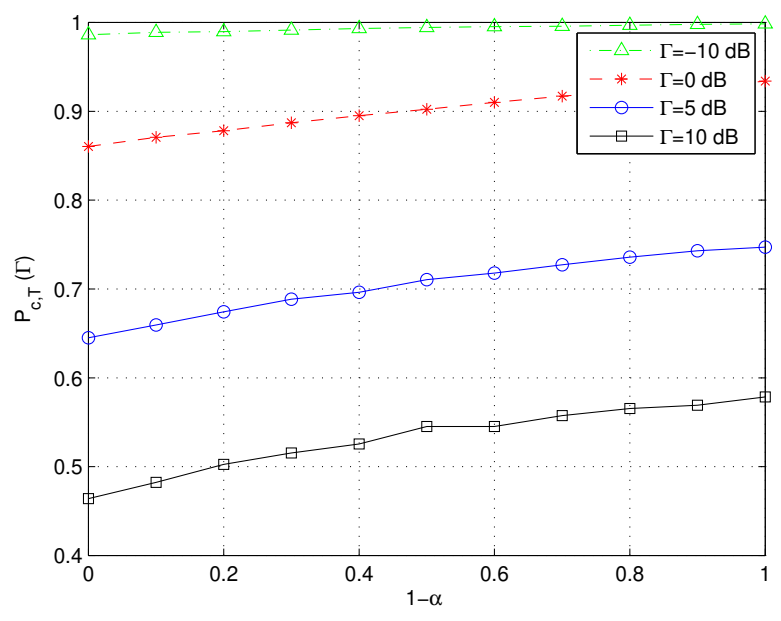

(a) SINR coverage probability versus $(1-\alpha)$

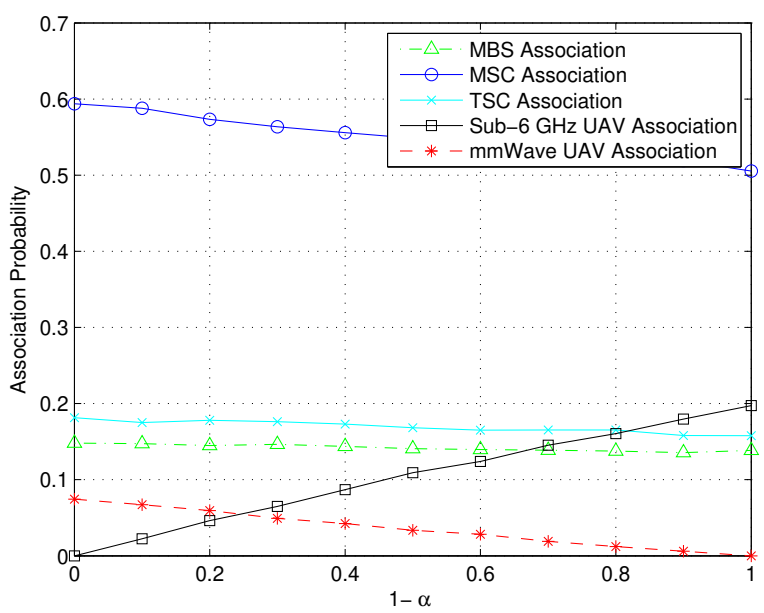

(b) Association probability versus $(1-\alpha)$

Fig. 8: Impact of proportion of sub-6GHz UAVs to mmWaveenabled UAVs, $(1-\alpha)$ on SINR coverage probability and Association probability with $\lambda_{1}=4 \times 10^{-6} \mathrm{BS} / \mathrm{m}^{2}, \lambda_{2}=$ $10 \times \lambda_{1} \mathrm{BS} / \mathrm{m}^{2}, \lambda_{3}=1 \times \lambda_{2} \mathrm{BS} / \mathrm{m}^{2}, \lambda_{4}=10 \times \lambda_{1} \mathrm{BS} / \mathrm{m}^{2}$, $\psi_{1}=\psi_{2}=0 \mathrm{~dB}, \psi_{3}=20 \mathrm{~dB}, \psi_{4}=10 \mathrm{~dB}$.

$\mathrm{GHz}$ UAVs, there is a decrease of about $40 \%$ users in coverage. Fig. $8 \mathrm{~b}$ shows the effect of user association probability versus proportion of sub- $6 \mathrm{GHz}$ UAVs to mmWave-enabled UAVs in the HetNet. From Fig. 8b, it is observed that for an increase in sub-6 GHz UAVs in the HetNet, there is an increase in the user association with sub-6 GHz UAVs. If we increase sub- $6 \mathrm{GHz}$ UAVs in the HetNet from $0 \%$ to $50 \%$, we can see that $10 \%$ more users are now associated with sub- $6 \mathrm{GHz}$ UAVs. As we increase the proportion of sub- $6 \mathrm{GHz}$ UAVs in HetNet, users associated with mmWave UAVs start to decrease. It can be concluded from Fig. 8 that an increase in the number of sub$6 \mathrm{GHz}$ UAVs in the HetNet results in more users connecting with sub-6 GHz UAVs and a better SINR coverage for users associated with sub-6 GHz UAVs than users connected with mmWave UAVs.

The trend of rate coverage probability versus proportion

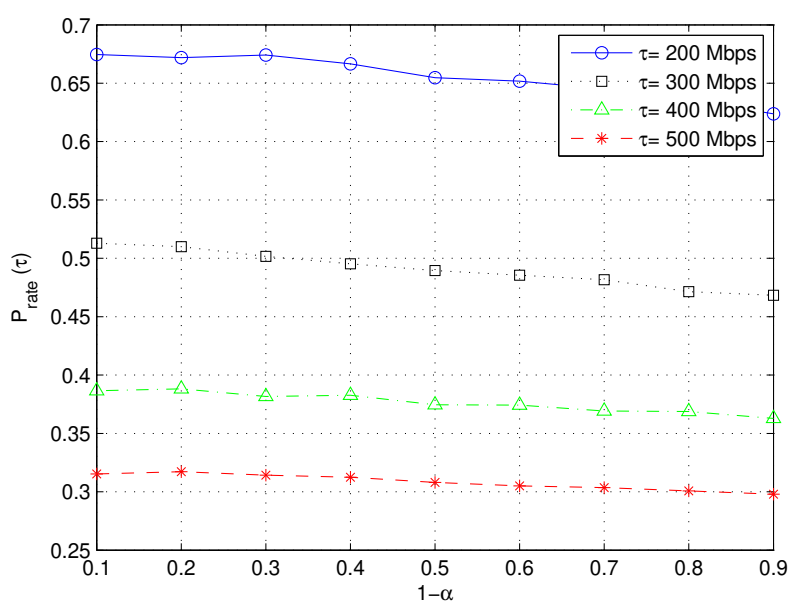

Fig. 9: Rate coverage probability versus proportion of sub6GHz UAVs to mmWave-enabled UAVs, $1-\alpha$ with $\lambda_{1}=4 \times$ $10^{-6} \mathrm{BS} / \mathrm{m}^{2}, \lambda_{2}=10 \times \lambda_{1} \mathrm{BS} / \mathrm{m}^{2}, \lambda_{3}=1 \times \lambda_{2} \mathrm{BS} / \mathrm{m}^{2}, \lambda_{4}=$ $10 \times \lambda_{1} \mathrm{BS} / \mathrm{m}^{2}, \psi_{1}=\psi_{2}=0 \mathrm{~dB}, \psi_{3}=20 \mathrm{~dB}, \psi_{4}=10 \mathrm{~dB}$.

of sub-6 GHz UAVs to mmWave-enabled UAVs for different data rate thresholds is shown in Fig. 9. It can be observed from Fig. 9, that an increase in the sub-6 GHz UAVs in the HetNet results in a lesser rate coverage probability. This decrease is due to the availability of lesser bandwidth at sub-6 $\mathrm{GHz}$ UAVs. For a rate threshold of $300 \mathrm{Mbps}$, we can see a decline of around $5 \%$ in rate coverage probability for an increase in proportion of sub- $6 \mathrm{GHz}$ UAVs in the HetNet by $80 \%$ by adjusting the tuning paramter, $1-\alpha$, from 0.1 to 0.9 . Fig. 9 also depicts the effect of various rate thresholds on rate coverage probability. By increasing the rate thresholds from $200 \mathrm{Mbps}$ to $500 \mathrm{Mbps}$, we can see the number of users attaining higher data rates decrease significantly. For an equal number of sub- $6 \mathrm{GHz}$ and mmWave UAVs in the HetNet $(1-\alpha=0.5)$, the rate coverage probability drops by around $34 \%$ if we increase the rate threshold $\tau$ from $200 \mathrm{Mbps}$ to 500 Mbps. It can be concluded from Fig. 8 and Fig. 9 that an increase in sub-6 GHz UAVs in the HetNet results in a better SINR coverage probability on the expense of lower achievable data rates while mmWave UAVs provide better rate coverage probability on the expense of lower SINR coverage probability.

\section{CONCLUSIONS}

In this paper, we considered a multi-tier HetNet composed of mmWave and THz-enabled aerial and terrestrial BSs and derived an analytical model for its coverage analysis. A tractable approach was developed to derive the SINR coverage probability for each tier using stochastic geometry tools. The results highlighted the impacts of different base station densities, biasness, transmit powers and available bandwidths on user association, SINR and rate coverage probabilities. We have shown that increasing the mmWave and THz-enabled cell densities and increasing the bias factors of these tiers result in meeting the QoS requirements of high data rates for the users. It has also been found that offloading the users from sub-6 $\mathrm{GHz}$ to mmWave and $\mathrm{THz}$ frequencies will result in a lower 
SINR coverage probability due to high propagation losses at high frequencies but the high available bandwidths at these frequencies significantly counters this loss by providing very high data rates to users. In future, the study can be extended to optimize different network parameters, i.e., number of BSs, UAV heights and transmit powers to meet various QoS requirements. UAVs face challenges with respect to its flight time and energy consumption. This work can be extended to study the effects of flight time and energy consumption on the coverage and rate performance in a HetNet. Massive MIMO can also be incorporated at the MBS and the effects of increased antenna density on coverage and rate performance can be analyzed. In $\mathrm{THz}$ tier, the effects of beamforming on the coverage and rate performance can also be investigated. .

\section{APPENDIX A DERIVATION OF LEMMA 1}

The 2-D PPP null probability can be used to evaluate the probability $\mathbb{P}\left[R_{n}>\left(\frac{\widetilde{P}_{n} G_{n} x_{i}^{\beta_{i}}}{\widetilde{P}_{i} G_{i}}\right)^{1 / \beta_{n}}\right]$.

$\kappa$ in (17) can be evaluated as follows,

$$
\begin{aligned}
\kappa & =\prod_{n=1, n \neq i}^{4} \mathbb{P}\left[\text { No BS closer than }\left(\frac{\widetilde{P}_{n} G_{n} x^{\beta_{i}}}{\widetilde{P}_{i} G_{i}}\right)^{1 / \beta_{n}} \text { in } n^{\text {th }} \text { tier }\right] \\
& =\exp \left\{-\pi \lambda_{1}\left(\frac{\widetilde{P}_{1} x_{i}}{\widetilde{P}_{i}}\right)^{2 / \beta_{1}}\right\}+\exp \left\{-2 \pi \lambda_{2} \Upsilon(x)\right\} \\
& +\exp \left\{-2 \pi \lambda_{3} T(x)\right\}+\exp \left\{-2 \pi \lambda_{4} U(x)\right\}, \\
& f_{X_{i}}(x)=2 \pi \lambda_{i} \operatorname{xexp}\left\{-\pi \lambda_{i} x^{2}\right\},
\end{aligned}
$$

For the MSC tier, the association of a typical user is based on link length as the transmit power is assumed to be same for all links in the mmWave tier.

For MSC we assume that the tiers of LoS and NLoS BSs are independent, so

$$
\begin{aligned}
& S_{x}=\mathbb{P}\left[P_{r, i}>P_{r, 2}\right] \\
& =\mathbb{P}\left[P_{r, i}>\widetilde{P}_{2} G x^{-\beta_{L}}\right] \mathbb{P}\left[P_{r, i}>\widetilde{P}_{2} G x^{-\beta_{N}}\right] \\
& =\mathbb{P}\left[x>\left(\frac{\widetilde{P}_{2} G}{\widetilde{P}_{i}}\right)^{\frac{1}{\beta_{L}}} x^{\frac{\beta_{i}}{\beta_{L}}}\right] \mathbb{P}\left[x>\left(\frac{\widetilde{P}_{2} G}{\widetilde{P}_{i}}\right)^{\frac{1}{\beta_{N}}} x^{\frac{\beta_{i}}{\beta_{N}}}\right] \\
& e^{-2 \pi \lambda_{2}} \underbrace{\left(\int_{0}^{\Xi_{N}(x)} t \mathfrak{P}_{L o S}(t) d t+\int_{0}^{\Xi_{L}(x)} t\left(1-\mathfrak{P}_{L o S}(t)\right)\right)}_{\Upsilon(x)} d t
\end{aligned}
$$

where $\Xi_{N}(x)=\left(\frac{\widetilde{P}_{2} G}{\widetilde{P}_{i}}\right)^{\frac{1}{\beta_{L}}} x^{\frac{\beta_{i}}{\beta_{L}}}$ and $\Xi_{L}(x)=\left(\frac{\widetilde{P}_{2} G}{\widetilde{P}_{i}}\right)^{\frac{1}{\beta_{N}}} x^{\frac{\beta_{i}}{\beta_{N}}}$ and (a) is derived from the null probability and $\mathfrak{P}_{L o S}(t)$ is the function of the LoS probability.
For the UAV mmWave tier,

$$
\begin{aligned}
W_{r}= & \mathbb{P}\left[P_{r, i}>P_{r, 4}\right] \\
= & \mathbb{P}\left[P_{r, i}>\widetilde{P}_{4} G r^{-\beta_{L}}\right] \mathbb{P}\left[P_{r, i}>\widetilde{P}_{4} G r^{-\beta_{N}}\right] \\
= & \left.\mathbb{P}\left[r>\widetilde{\widetilde{P}}_{\widetilde{P}_{i}}\right)^{\frac{1}{\beta_{L}}} x^{\frac{\beta_{i}}{\beta_{L}}}\right] \mathbb{P}\left[r>\left(\frac{\widetilde{P}_{4} G}{\widetilde{P}_{i}}\right)^{\frac{1}{\beta_{N}}} x^{\left.\frac{\beta_{i}}{\beta_{N}}\right]}\right. \\
& -2 \pi \alpha \lambda_{4} \underbrace{\left(\int_{0}^{\xi_{N}(r)} t \mathcal{P}_{L o S}(t) d t+\int_{0}^{\xi_{L}(r)} t\left(1-\mathcal{P}_{L o S}(t)\right)\right)}_{U(x)} d t \\
= & e
\end{aligned}
$$

where $\xi_{N}(r)=\left(\frac{\widetilde{P_{4}} G}{\widetilde{P}_{i}}\right)^{\frac{1}{\beta_{L}}} x^{\frac{\beta_{i}}{\beta_{L}}}$ and $\xi_{L}(r)=\left(\frac{\widetilde{P}_{4} G}{\widetilde{P}_{i}}\right)^{\frac{1}{\beta_{N}}} x^{\frac{\beta_{i}}{\beta_{N}}}$ where $r=\sqrt{x^{2}+h_{t}^{2}}$ and $\mathcal{P}_{L o S}(t)$ is the LoS probability function.

The probability that a user associates with $\mathrm{THz}$ BS can be evaluated as follows

$$
\begin{aligned}
& Y_{x}=\mathbb{P}\left[P_{r, i}>P_{r, 3}\right] \\
& =\mathbb{P}\left[P_{r, i}>\widetilde{P}_{3} \exp \left(-k\left(f_{c, 3}\right) x\right) x^{\left.-\beta_{L}\right]}\right. \\
& \mathbb{P}\left[P_{r, i}>\widetilde{P}_{3} \exp \left(-k\left(f_{c, 3}\right) x\right) x^{-\beta_{N}}\right] \\
& \left.=\mathbb{P}\left[x>\left(\frac{\widetilde{P}_{3} \exp \left(-k\left(f_{c, 3}\right) x\right)}{\widetilde{P}_{i}}\right)^{\frac{1}{\beta_{3}}} x^{\left.\frac{\beta_{i}}{\beta_{3}}\right]}\right)^{\frac{1}{\beta_{N}}} x^{\frac{\beta_{i}}{\beta_{N}}}\right] \\
& \mathbb{P}\left[x>(\frac{\widetilde{P}_{3} \exp \left(-k\left(f_{c, 3}\right) x\right)}{\widetilde{P}_{i}} \underbrace{\left.t_{0}^{\Delta_{L}(x)} t\left(1-\mathfrak{P}_{L o S}(t)\right)\right)}_{T(x)} d t\right.
\end{aligned}
$$

where $\Delta_{N}(x)=\left(\frac{\widetilde{P}_{3} \exp \left(-k\left(f_{c, 3}\right) x\right) G}{\widetilde{P}_{i}}\right)^{\frac{1}{\beta_{L}}} x^{\frac{\beta_{i}}{\beta_{L}}}$ and $\Delta_{L}(x)=\left(\frac{\widetilde{P}_{3} \exp \left(-k\left(f_{c, 3}\right) x\right) G}{\widetilde{P}_{i}}\right)^{\frac{1}{\beta_{N}}} x^{\frac{\beta_{i}}{\beta_{N}}}$

where $\mathfrak{P}_{L o S}(t)$ is the LoS probability function.

\section{APPENDIX B \\ DERIVATION OF LEMMA 2}

The conditional coverage probability, $P_{c}^{1}\left(\Gamma_{1}, x\right)$, can be evaluated as, 


$$
\begin{aligned}
P_{C}^{1}\left(\Gamma_{1}, x\right) & =\operatorname{Pr}\left(\frac{\widetilde{P}_{1} h_{M} x^{-\beta_{1}}}{\sigma_{1}^{2}+I_{1}}>\Gamma_{1}\right) \\
& =\operatorname{Pr}\left(h_{M}>\frac{\Gamma_{1} x^{\beta_{1}}\left(\sigma_{1}^{2}+I_{1}\right)}{\widetilde{P}_{1}}\right) \\
& \stackrel{(a)}{=} \exp \left(\frac{-\Gamma_{1} x^{\beta_{1}} \sigma_{1}^{2}}{\widetilde{P}_{1}}\right) \mathbb{E}_{I_{1}}\left[\exp \left(\frac{-\Gamma_{1} x^{\beta_{1}} I_{1}}{\widetilde{P}_{1}}\right)\right] \\
& =\exp \left(\frac{-\Gamma_{1} x^{\beta_{1}} \sigma_{1}^{2}}{\widetilde{P}_{1}}\right) \mathbb{E}_{I_{1}}\left[\mathcal{L}_{I_{1}}\left(\frac{\Gamma_{1} x^{\beta_{1}}}{\widetilde{P}_{1}}\right)\right],
\end{aligned}
$$

where (a) follows from the exponential distribution of $h_{M}$. By substituting $P_{c}^{1}\left(\Gamma_{1}, x\right)$ and $f_{X_{1}}(x)$ into (18), we can compute the SINR coverage probability.

\section{APPENDIX C}

\section{DERIVATION OF LEMMA 3}

The conditional coverage probability that a user is associated with $\mathrm{BS}$ in $\phi_{v}$ where $v \in\{L, N\}$, can be found as

$$
P_{c}^{2, v}=\delta_{2, v} \int_{0}^{\infty} \mathbb{P}\left[\frac{\widetilde{P}_{2} G_{r}^{m m} G_{t}^{m m} h_{o, m m} x^{-\beta_{v}}}{\sigma^{2}+I_{L}+I_{N}+I_{L_{U}}+I_{N_{U}}}>\Gamma_{2}\right] f_{v}(x) d x,
$$

where $I_{L}$ and $I_{N}$ are the $\operatorname{LoS}$ and NLoS interferences from MSCs and $I_{L U}$ and $I_{N U}$ are the LoS and NLoS interferences from UAVs. The LoS and NLoS link probabilities of the user are given as,

$$
\begin{aligned}
\delta_{2, L} & =2 \pi \lambda_{2} \int_{0}^{\infty} x \mathfrak{P}_{L o S}(x) \exp \left(-2 \pi \lambda_{2} Z(x)-A(x)-B(x)\right. \\
& -C(x)) d x
\end{aligned}
$$

where

$$
\begin{gathered}
Z(x)=\int_{0}^{x} t \mathfrak{P}_{L o S}(t) d t+\int_{0}^{x^{\beta_{L}} / \beta_{N}} t\left(1-\mathfrak{P}_{L o S}(t)\right) d t \\
A(x)=\pi \lambda_{1}\left(\frac{\widetilde{P}_{1} x_{L}}{\widetilde{P}_{2}}\right)^{2 / \beta_{1}} \\
B(x)=2 \pi \lambda_{3} T(x) \\
C(x)=2 \pi \alpha \lambda_{4} U(x)
\end{gathered}
$$

and

$$
\begin{aligned}
\delta_{2, N} & =2 \pi \lambda_{2} \int_{0}^{\infty} x \mathfrak{P}_{L o S}(x) \exp \left(-2 \pi \lambda_{2} Z_{N}(x)-A_{N}(x)-B_{N}(x)\right. \\
& \left.-C_{N}(x)\right) d x
\end{aligned}
$$

where

$$
Z_{N}(x)=\int_{0}^{x} t\left(1-\mathfrak{P}_{L o S}(t)\right) d t+\int_{0}^{x^{\beta_{N} / \beta_{L}}} t \mathfrak{P}_{L o S}(t) d t,
$$

$$
\begin{gathered}
A_{N}(x)=\pi \lambda_{1}\left(\frac{\widetilde{P}_{1} x^{\beta_{N}}}{\widetilde{P}_{2}}\right)^{2 / \beta_{1}}, \\
B_{N}(x)=2 \pi \lambda_{3} T(x), \\
C_{N}(x)=2 \pi \alpha \lambda_{4} U(x),
\end{gathered}
$$

The PDF of the distance to the LoS BS, given that user is associated with LoS small cell BS is given by,

$$
\begin{gathered}
f_{L}^{\wedge}(x)=\left(2 \pi \lambda_{2} x \mathfrak{P}_{L o S}(x) \exp \left(-2 \pi \lambda_{2} \int_{0}^{x}\left(\mathfrak{P}_{L o S}(t)\right) t d t\right)\right. \\
\left.\exp \left(-2 \pi \lambda_{2} \int_{0}^{\Xi_{L}(x)}\left(1-\mathfrak{P}_{L o S}(t)\right) t d t\right)\right) / \delta_{2, L},
\end{gathered}
$$

where $\mathfrak{P}_{L o S}(x)$ represents the LoS probability function. Similarly for the NLoS BS,

$$
\begin{aligned}
& f_{N}^{\wedge}(x)=\left(2 \pi \lambda _ { 2 } x ( 1 - \mathfrak { P } _ { L o S } ( x ) ) \operatorname { e x p } \left(-2 \pi \lambda_{2} \int_{0}^{x}\left(1-\mathfrak{P}_{L o S}(t)\right) t d t\right.\right. \\
&\left.\exp \left(-2 \pi \lambda_{2} \int_{0}^{\Xi_{N}(x)}\left(\mathfrak{P}_{L o S}(t)\right) t d t\right)\right) / \delta_{2, N},
\end{aligned}
$$

where $\Xi_{L}(x)$ and $\Xi_{N}(x)$ are given in Appendix A. Now $I_{L}=P_{2} \sum_{i \in \phi_{m m . L}^{\wedge}} G_{V} h_{i, m m} x^{-\beta_{L}}$ and $I_{N}=P_{2} \sum_{i \in \phi_{m m, N}^{\wedge}} G_{V} h_{i, m m} x^{-\beta_{N}}$ are the interference from LoS and NLoS BSs, respectively and $I_{L U}=P_{4} \sum_{i \in \phi_{u, L}^{\wedge}} G_{e} h_{i, u} r^{-\beta_{L}}$ and $I_{N U}=P_{4} \sum_{i \in \phi_{u, N}^{\wedge}} G_{e} h_{i, u} r^{-\beta_{N}}$ are the interference from LoS and NLoS UAVs. We assumed Nakagami fading, therefore $h_{o, m}$ is a normalized gamma random variable with parameters $\Omega_{w}$.

$P_{c}^{2, v}=$

$\mathbb{P}\left[\widetilde{P}_{2} G_{r}^{m m} G_{t}^{m m} h_{o, m m} x^{-\beta_{v}}>\Gamma_{2}\left(\sigma_{2}^{2}+I_{L}+I_{N}+I_{L U}+I_{N U}\right)\right]$,

$=\mathbb{P}\left[h_{o, m m}>\frac{x^{\beta_{v}} \Gamma_{2}\left(\sigma_{2}^{2}+I_{L}+I_{N}+I_{L U}+I_{N U}\right)}{\widetilde{P}_{2} G_{r}^{m m} G_{t}^{m m}}\right]$,

$\stackrel{(a)}{=} 1-$

$\mathbb{E}\left[\left(1-\exp \left(\frac{-\rho_{w} x^{\beta_{v}} \Gamma_{2}\left(\sigma_{2}^{2}+I_{L}+I_{N}+I_{L U}+I_{N U}\right)}{\widetilde{P}_{2} G_{r}^{m m} G_{t}^{m m}}\right)\right)^{\Omega_{w}}\right]$,

$\stackrel{(b)}{=} \sum_{i=1}^{\Omega_{w}}(-1)^{i+1}\left(\begin{array}{c}\Omega_{w} \\ i\end{array}\right)$

$\mathbb{E}\left[\exp \left(\frac{-i \rho_{w} x^{\beta_{v}} \Gamma_{2}\left(\sigma_{2}^{2}+I_{L}+I_{N}+I_{L U}+I_{N U}\right)}{\widetilde{P}_{2} G_{r}^{m m} G_{t}^{m m}}\right)\right]$,

$\stackrel{(c)}{=} \sum_{i=1}^{\Omega_{w}}(-1)^{i+1}\left(\begin{array}{c}\Omega_{w} \\ i\end{array}\right) e^{\frac{-i \rho_{w} x^{\beta_{v}} \Gamma_{2} \sigma_{2}^{2}}{\widetilde{P}_{2} G_{r}^{m m} G_{t}^{m m}}}$

$\mathbb{E}_{I_{L}}\left[\exp \left(\frac{-i \rho_{w} x^{\beta_{v}} \Gamma_{2} I_{L}}{\widetilde{P}_{2} G_{r}^{m m} G_{t}^{m m}}\right)\right] \mathbb{E}_{I_{N}}\left[\exp \left(\frac{-i \rho_{w} x^{\beta_{v}} \Gamma_{2} I_{N}}{\widetilde{P}_{2} G_{r}^{m m} G_{t}^{m m}}\right)\right]$

$\mathbb{E}_{I_{L U}}\left[\exp \left(\frac{-i \rho_{w} x^{\beta_{v}} \Gamma_{2} I_{L U}}{\widetilde{P}_{2} G_{r}^{m m} G_{t}^{m m}}\right)\right] \mathbb{E}_{I_{N U}}\left[\exp \left(\frac{-i \rho_{w} x^{\beta_{v}} \Gamma_{2} I_{N U}}{\widetilde{P}_{2} G_{r}^{m m} G_{t}^{m m}}\right)\right]$, 
where (a) follows from [27] and $\rho_{w}=\Omega_{w}\left(\Omega_{w} !\right)^{-1 / \Omega_{w}}, w \in$ $\left\{l_{m}, n_{m}\right\}$, (b) is derived using binomial expansion and (c) is obtained using the independence of $\phi_{L}$ and $\phi_{N}$ PPPs. Now $E_{I_{L}}$ is given as,

$$
\begin{aligned}
& \mathbb{E}_{I_{L}}\left[\exp \left(\frac{-i \rho_{w} x^{\beta_{v}} \Gamma_{2} I_{L}}{\widetilde{P}_{2} G_{r}^{m m} G_{t}^{m m}}\right)\right]= \\
& \mathbb{E}_{I_{L}}\left[\exp \left(\frac{-i \rho_{w} x^{\beta_{L}} \Gamma_{2} \sum_{i \in \phi_{m, L}^{\wedge}} G_{V} h_{i, m m} x^{-\beta_{L}}}{G_{r}^{m m} G_{t}^{m m}}\right)\right], \\
& \stackrel{(d)}{=} \exp \left(-2 \pi \lambda_{2} \sum_{i=1}^{4} p_{i} \int_{x}^{\infty}\left(1-E_{g}\left[e^{\left.-i \rho_{l m} x^{\beta_{L}} \Gamma_{2} g \hat{a}_{i} t^{-\beta_{L}}\right]}\right)\right.\right. \\
& \left.\left(\mathfrak{P}_{L o S}(t)\right) t d t\right), \\
& \stackrel{(e)}{=} \prod_{i=1}^{4} \exp \left(-2 \pi \lambda_{2} p_{i} \int_{x}^{\infty}\left(1-\frac{1}{\left(i \rho_{l_{m}} x^{\beta_{L}} \Gamma_{2} \Omega_{l_{m}}^{-1} \hat{a}_{i} t^{-\beta_{L}}\right)^{\Omega_{l_{m}}}}\right)\right. \\
& \left.\left(\mathfrak{P}_{L o S}(t)\right) t d t\right)
\end{aligned}
$$

where Laplace transform of $\phi_{L}$ gives us $60(d)$. In $60(d), \Omega_{l_{m}}$ is the parameter of a normalized gamma random variable representing channel gain $g, \hat{a}_{i}$ represents the gain $a_{i}$ which is normalized by $G_{r}^{m m} G_{t}^{m m}$, where (7) contains the parameters $a_{i}$ and $p_{i} .60(e)$ is found by computing the moment generating functional of $g . \mathbb{E}_{I_{N}}$ can be found in a similar way as $\mathbb{E}_{I_{L}}$. Final expression for $\mathbb{E}_{I_{N}}$ is given as,

$$
\begin{aligned}
& \mathbb{E}_{I_{N}}\left[\exp \left(\frac{-i \rho_{w} x^{\beta_{v}} \Gamma_{2} I_{N}}{\widetilde{P}_{2} G_{r}^{m m} G_{t}^{m m}}\right)\right]= \\
& \prod_{i=1}^{4} \exp \left(-2 \pi \lambda_{2} p_{i} \int_{\Xi_{L}(x)}^{\infty}\left(1-\frac{1}{\left(i \rho_{l_{m}} x \beta_{L} \Gamma_{2} \Omega_{n_{m}}^{-1} \hat{a}_{i} t^{-\beta_{N}}\right)^{\Omega_{n_{m}}}}\right)\right. \\
& \left.\left(1-\mathfrak{P}_{L o S}(t)\right) t d t\right),
\end{aligned}
$$

where $\Omega_{n}$ is parameter of a normalized gamma random variable representing channel gain. Similarly, $\mathbb{E}_{I_{L U}}$ is given as

$$
\begin{aligned}
& \mathbb{E}_{I_{L U}}\left[\exp \left(\frac{-i \rho_{w} x^{\beta_{v}} \Gamma_{2} I_{L U}}{\widetilde{P}_{2} G_{r}^{m m} G_{t}^{m m}}\right)\right]= \\
& \prod_{i=1}^{4} \exp \left(-2 \pi \alpha \lambda_{4} p_{i} \int_{x}^{\infty}\left(1-\frac{1}{\left(i \rho_{l_{m}} x^{\beta_{L}} \Gamma_{2} \Omega_{l_{m}}^{-1} \hat{a}_{i} t^{-\beta_{L}}\right)^{\Omega_{l_{m}}}}\right)\right. \\
& \left.\left(\mathfrak{P}_{L o S}(t)\right) t d t\right),
\end{aligned}
$$

and $\mathbb{E}_{I_{N U}}$ is given as,

$$
\begin{aligned}
& \mathbb{E}_{I_{N U}}\left[\exp \left(\frac{-i \rho_{w} x^{\beta_{v}} \Gamma_{2} I_{N U}}{\widetilde{P}_{2} G_{r}^{m m} G_{t}^{m m}}\right)\right]= \\
& \prod_{i=1}^{4} \exp \left(-2 \pi \alpha \lambda_{4} p_{i} \int_{\xi_{L}(x)}^{\infty}\left(1-\frac{1}{\left(i \rho_{l_{m}} x \beta_{L} \Gamma_{2} \Omega_{n_{m}}^{-1} \hat{a}_{i} t^{-\beta_{N}}\right)^{\Omega_{n_{m}}}}\right)\right. \\
& \left.\left(1-\mathcal{P}_{L o S}(t)\right) t d t\right)
\end{aligned}
$$

where $\mathcal{P}_{L o S}(x)$ is the LoS probability between user and UAV and $x$ denotes the horizontal distance between the user and UAV projection on ground. By combining interference terms using linearity of integrals for $v=L$, we obtain the coverage probability for LoS. For $v=N$, coverage probability can be obtained by following similar steps.

\section{APPENDIX D DERIVATION OF LEMMA 6}

The achievable data rate for a user connected with $i^{\text {th }}$ tier is given as,

$$
\begin{aligned}
R_{i} & =\mathbb{P}\left(R_{i}>\Gamma_{i}\right)=\mathbb{P}\left(\log _{2}\left(1+\operatorname{SINR}_{i}\right)>\Gamma_{i}\right) \\
& =\mathbb{P}\left(\operatorname{SINR}_{i}>2^{\Gamma_{i}}-1\right) \\
& =\frac{1}{\ln 2} \int_{0}^{\infty} \mathbb{P}\left(\operatorname{SINR}_{i}>2^{\Gamma_{i}}-1\right) \\
& =\frac{1}{\ln 2} \int_{0}^{\infty} \frac{P_{c}^{i}\left(\Gamma_{i}\right)}{1+\Gamma_{i}} d \Gamma_{i},
\end{aligned}
$$

where $P_{c}^{i}$ is the coverage probability of tier $i$ and $\Gamma_{i}$ is the SINR threshold of tier $i$.

\section{ACKNOWLEDGEMENT}

The authors are grateful to the Deanship of Scientific Research at King Saud University, Riyadh, Saudi Arabia for funding this work through the Vice Deanship of Scientific Research Chairs: Chair of Pervasive and Mobile Computing.

\section{REFERENCES}

[1] Y. Niu, Y. Li, D. Jin, L. Su, and A. V. Vasilakos, "A survey of millimeter wave communications (mmwave) for 5G: opportunities and challenges," Wireless networks, vol. 21, no. 8, pp. 2657-2676, 2015.

[2] Y. Lu and X. Zheng, "6G: A survey on technologies, scenarios, challenges, and the related issues," Journal of Industrial Information Integration, p. 100158, 2020.

[3] Z. Ullah, F. Al-Turjman, and L. Mostarda, "Cognition in UAV-aided 5G and beyond communications: A survey," IEEE Transactions on Cognitive Communications and Networking, vol. 6, no. 3, pp. 872-891, 2020.

[4] I. F. Akyildiz, J. M. Jornet, and C. Han, "Terahertz band: Next frontier for wireless communications," Physical Communication, vol. 12, pp. $16-32,2014$.

[5] I. F. Akyildiz, C. Han, and S. Nie, "Combating the distance problem in the millimeter wave and terahertz frequency bands," IEEE Communications Magazine, vol. 56, no. 6, pp. 102-108, 2018.

[6] Z. Xiao, Q. Yang, J. Huang, Z. Huang, W. Zhou, Y. Gao, R. Shu, and Z. He, "Terahertz communication windows and their point-to-point transmission verification," Applied Optics, vol. 57, no. 27, pp. 76737680, 2018.

[7] C. Chaccour, M. N. Soorki, W. Saad, M. Bennis, P. Popovski, and M. Debbah, "Seven defining features of terahertz (THz) wireless systems: A fellowship of communication and sensing," arXiv preprint arXiv:2102.07668, 2021. 
[8] C. Wang and Y. J. Chun, "Stochastic geometry modeling and analysis for THz-mmWave hybrid IoT networks," arXiv preprint arXiv:2103.11674, 2021.

[9] J. G. Andrews, A. K. Gupta, and H. S. Dhillon, "A primer on cellular network analysis using stochastic geometry," arXiv preprint arXiv:1604.03183, 2016.

[10] M. Mozaffari, W. Saad, M. Bennis, and M. Debbah, "Unmanned aerial vehicle with underlaid device-to-device communications: Performance and tradeoffs," IEEE Transactions on Wireless Communications, vol. 15 , no. 6, pp. 3949-3963, 2016.

[11] G. Fontanesi, A. Zhu, and H. Ahmadi, "Outage analysis for millimeterwave fronthaul link of UAV-aided wireless networks," IEEE Access, vol. 8, pp. $111693-111706,2020$.

[12] M. Boschiero, M. Giordani, M. Polese, and M. Zorzi, "Coverage analysis of UAVs in millimeter wave networks: A stochastic geometry approach," arXiv preprint arXiv:2003.01391, 2020.

[13] W. Yi, Y. Liu, E. Bodanese, A. Nallanathan, and G. K. Karagiannidis, "A unified spatial framework for UAV-aided mmwave networks," IEEE Transactions on Communications, vol. 67, no. 12, pp. 8801-8817, 2019.

[14] X. Wang and M. C. Gursoy, "Coverage analysis for energy-harvesting UAV-assisted mmwave cellular networks," IEEE Journal on Selected Areas in Communications, vol. 37, no. 12, pp. 2832-2850, 2019.

[15] J. Sayehvand and H. Tabassum, "Interference and coverage analysis in coexisting rf and dense terahertz wireless networks," IEEE Wireless Communications Letters, 2020.

[16] K. Ntontin and C. Verikoukis, "Toward the performance enhancement of microwave cellular networks through THz links," IEEE Transactions on Vehicular Technology, vol. 66, no. 7, pp. 5635-5646, 2017.

[17] J. Kokkoniemi, J. Lehtomäki, and M. Juntti, "Stochastic geometry analysis for mean interference power and outage probability in $\mathrm{THz}$ networks," IEEE Transactions on Wireless Communications, vol. 16, no. 5, pp. 3017-3028, 2017.

[18] S. A. R. Naqvi, S. A. Hassan, H. Pervaiz, and Q. Ni, "Drone-aided communication as a key enabler for $5 \mathrm{G}$ and resilient public safety networks," IEEE Communications Magazine, vol. 56, no. 1, pp. 36-42, 2018.

[19] A. Al-Hourani, S. Kandeepan, and S. Lardner, "Optimal lap altitude for maximum coverage," IEEE Wireless Communications Letters, vol. 3, no. 6, pp. 569-572, 2014.

[20] M. K. Shehzad, S. A. Hassan, A. Mahmood, and M. Gidlund, "On the association of small cell base stations with UAVs using unsupervised learning," in 2019 IEEE 89th Vehicular Technology Conference (VTC2019-Spring), pp. 1-5.

[21] M. A. Jan, S. A. Hassan, and H. Jung, "Qos-based performance analysis of mmwave UAV-assisted 5G hybrid heterogeneous network," in 2019 IEEE Global Communications Conference (GLOBECOM). IEEE, 2019, pp. 1-6.

[22] I. Atzeni, J. Arnau, and M. Kountouris, "Downlink cellular network analysis with los/nlos propagation and elevated base stations," IEEE Transactions on Wireless Communications, vol. 17, no. 1, pp. 142-156, 2018.

[23] V. V. Chetlur and H. S. Dhillon, "Downlink coverage analysis for a finite 3-d wireless network of unmanned aerial vehicles," IEEE Transactions on Communications, vol. 65, no. 10, pp. 4543-4558, 2017.

[24] B. Galkin, J. Kibiłda, and L. A. DaSilva, "A stochastic model for UAV networks positioned above demand hotspots in urban environments," IEEE Transactions on Vehicular Technology, vol. 68, no. 7, pp. 69856996, 2019.

[25] M. S. Omar, S. A. Hassan, H. Pervaiz, Q. Ni, L. Musavian, S. Mumtaz, and O. A. Dobre, "Multiobjective optimization in 5G hybrid networks," IEEE Internet of Things Journal, vol. 5, no. 3, pp. 1588-1597, 2018.

[26] A. Umer, S. A. Hassan, H. Pervaiz, L. Musavian, Q. Ni, and M. A. Imran, "Secrecy spectrum and energy efficiency analysis in massive mimo-enabled multi-tier hybrid hetnets," IEEE Transactions on Green Communications and Networking, vol. 4, no. 1, pp. 246-262, 2019.

[27] T. Bai and R. W. Heath, "Coverage and rate analysis for millimeter-wave cellular networks," IEEE Transactions on Wireless Communications, vol. 14, no. 2, pp. 1100-1114, 2015.

[28] M. R. Akdeniz, Y. Liu, M. K. Samimi, S. Sun, S. Rangan, T. S. Rappaport, and E. Erkip, "Millimeter wave channel modeling and cellular capacity evaluation," IEEE Journal on Selected Areas in Communications, vol. 32, no. 6, pp. 1164-1179, 2014.

[29] J. G. Andrews, T. Bai, M. N. Kulkarni, A. Alkhateeb, A. K. Gupta, and R. W. Heath, "Modeling and analyzing millimeter wave cellular systems," IEEE Transactions on Communications, vol. 65, no. 1, pp. 403-430, 2017.
[30] M. T. Dabiri, H. Safi, S. Parsaeefard, and W. Saad, "Analytical channel models for millimeter wave UAV networks under hovering fluctuations," IEEE Transactions on Wireless Communications, vol. 19, no. 4, pp. $2868-2883,2020$.

[31] S. T. Muntaha, S. A. Hassan, H. Jung, and M. S. Hossain, "Energy efficiency and hover time optimization in UAV-based hetnets," IEEE Transactions on Intelligent Transportation Systems, pp. 1-9, 2020.

[32] S. Kumar, S. Suman, and S. De, "Dynamic resource allocation in UAVenabled mmwave communication networks," IEEE Internet of Things Journal, 2020.

[33] L. S. Rothman, "The hitran 2008 molecular spectroscopic database," Journal of Quantitative Spectroscopy and Radiative Transfer, vol. 110, no. 9-10, pp. 533-572, 2009.

[34] B. Galkin, J. Kibilda, and L. A. DaSilva, "Backhaul for low-altitude UAVs in urban environments," in 2018 IEEE International Conference on Communications (ICC). IEEE, 2018, pp. 1-6. 